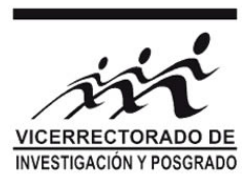

\title{
Análisis Comparativo de Datos Superficiales de Vientos Modelizados y Observados (Parte 1)
}

\author{
Miguel Saavedra ${ }^{* 1}$, Boris Dewite ${ }^{2}$, Lionel Renauld ${ }^{3}$, José Pasapera ${ }^{4}$ y César Jiménez ${ }^{1}$ \\ ${ }^{1}$ Universidad Nacional Mayor de San Marcos, Perú \\ ${ }^{2}$ Institut de Recherche pour le Développement, París, France \\ ${ }^{3}$ Laboratoire d'Etudes en Géophysique et Océanographie Spatiales, Toulouse, France \\ ${ }^{4}$ Instituto del Mar del Perú, Puno
}

Recibido 26 octubre 2018 - Aceptado 23 diciembre 2018

\begin{abstract}
En este trabajo se evaluó el realismo de las simulaciones de vientos superficiales realizados con el modelo numérico "Weather Research and Forecasting" (WRF) con datos obtenidos del satélite QuikSCAT (Quick Scatterometer). Se utilizan como parámetros de comparación las velocidades del viento, así como los esfuerzos producidos sobre la superficie del agua. El conocimiento de la configuración del viento sobre la superficie oceánica es importante ya que genera procesos como el afloramiento, la espiral y el bombeo de Ekman ("Ekman pumping"). La intensidad con las que se llevan a cabo estos procesos, determina la diversidad y riqueza biológica y pesquera de una región. Se analiza el comportamiento del viento en cuanto a sus velocidades horizontales, el esfuerzo producido por estas velocidades, que determinan el arrastre sobre una superficie libre y el rotacional del viento. Se obtienen correlaciones superiores a 0.6 en las áreas oceánicas, alejadas de la costa, mientras que para regiones costeras las correlaciones son menores a 0,4 . Palabras claves: Viento, esfuerzo, rotacional, Reanálisis, Circulación oceánica, circulación atmosférica.
\end{abstract}

\section{Comparative Analysis of Surface Data of Modeled and Observed Winds (Part 1)}

In the present work, the realism of the superficial wind simulations carried out with the model is evaluated emph "Weather Research and Forecasting" (WRF) is evaluated, with data obtained from the QuikSCAT (Quick Scatterometer) satellite. The meridional and zonal speeds of the wind are used as comparison parameters, as well as the stresses produced on the surface of the water. The knowledge of the configuration of the wind on the oceanic surface is important because it generates processes such as the upwelling, the spiral and the "Ekman pumping". The intensity with which these processes are carried out determines the diversity and biological and fishing richness of a region. The behavior of the wind is analyzed in terms of its horizontal speeds, the effort produced by these speeds, which determine the drag on a free surface and the rotational wind. Correlations greater than 0.6 are obtained in the oceanic areas, far from the coast, while for coastal regions the correlations are less than 0.4 .

Keywords: Reanalysis, atmospheric circulation, wind, stress, curl.

\section{Introducción}

La importancia de la circulación atmosférica, principalmente el viento, radica en que es el forzante principal para la circulación del océano y además es un factor que determina las características climáticas de una región. En la actualidad se conoce que anomalías de viento pueden forzar ondas oceánicas con longitudes de onda del orden de 13000 - $15000 \mathrm{~km}$ (tamaño de la cuenca del Océano Pacífico), que afectan la circulación oceánica y

\footnotetext{
*msaavedraj@unmsm.edu.pe
}

el clima [1], [2], pero también puede modificar la circulación oceánica regional, particularmente, en regiones de afloramiento, donde la variabilidad de viento es grande [3].

El afloramiento costero, sobre los sistemas de fronteras del Este, depende críticamente de las características de la circulación atmosférica que se dirige hacia el Ecuador, mientras que muchas características del patrón de corrientes costeras son forzadas por el esfuerzo local del viento, los modelos oceánicos regionales utilizan forzantes derivados de reanálisis o climatologías [4]. Sin embargo, 


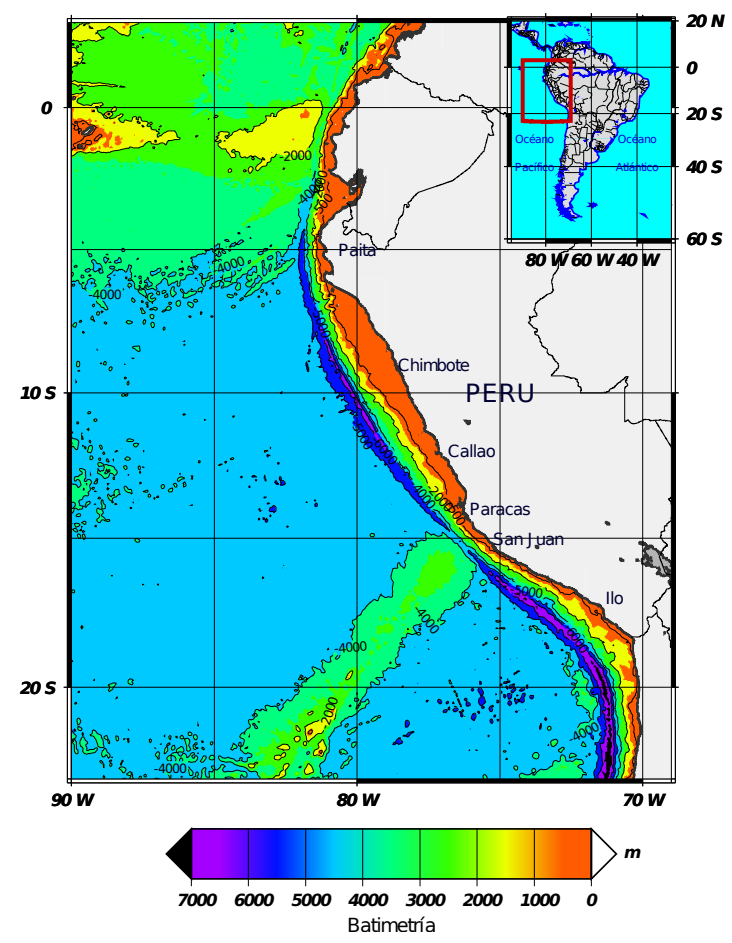

Figura 1: Topografía de Suramérica (elaborada con datos de ETOPO2).

este tipo de forzantes no resuelve las particularidades de la circulación atmosférica regional. En este estudio es un avance hacia el uso de datos de viento de alta resolución, provenientes de modelos atmosféricos, para forzar los modelos oceánicos regionales en el dominio del sistema de corrientes del Perú, o en otra área de interés.

En este trabajo, se realiza una comparación de los datos de observaciones satelitales de QuikSCAT con las salidas de un modelo numérico de circulación atmosférica regional, The Weather Research and Forecasting Model (WRF). Este modelo, es de última generación para predicción de las condiciones atmosféricas en la meso-escala diseñado para pronósticos operacionales e investigación atmosférica. El primer objetivo de este trabajo es determinar la habilidad del modelo WRF para reproducir la circulación atmosférica superficial para el periodo de estudio (año 2000). El segundo objetivo es diagnosticar cómo el modelo regional simula los gradientes horizontales del viento sobre la franja costera, donde no existen observaciones o tienen serias limitaciones, por el problemas de la zona ciega (Blind zone).

Se analizaron las características relevantes del momentum del forzante de la circulación oceánica y el afloramiento (rotacional del esfuerzo de viento, gradientes perpendiculares a la costa, etc). Para comparar la variabilidad de la circulación atmosférica modelada y observada, se utilizaron datos de viento para el año 2000 obtenidos de la base de datos internacionales QuikSCAT y datos de viento obtenidos de simulaciones mediante el modelo atmosférico WRF. El ámbito geográfico en el que se realizó el estudio es una parte del Océano Pacífico suroriental, entre los paralelos $23^{\circ} \mathrm{S}-3^{\circ} \mathrm{N}$ y meridianos $90^{\circ} \mathrm{O}-69^{\circ} \mathrm{O}$. En la Figura 1, se muestra la zona de estudio.

\section{Materiales y Métodos}

Para la elaboración de este trabajo, se realizaron simulaciones numéricas con el modelo atmosférico WRF, con datos correspondientes al año 2000, utilizando como condiciones de frontera los datos de reanálisis de NCEPNCAR (National Center for Environmental Prediction The National Center for Atmospheric Research). Se obtuvieron salidas horarias, de las componentes de viento, en 57 niveles verticales para el área de interés. Además se utilizaron datos de viento observados mediante el satélite QuikSCAT (Quick Scatterometer), los cuales son utilizados como referencia. Los datos de vientos superficiales fueron procesados para obtener promedios diarios, mensuales, bi-mensuales. También se calcularon los esfuerzos, el rotacional (de la velocidad del viento); asimismo, se calcularon correlaciones entre los datos de velocidad de QuikSCAT y del modelo WRF y RMS (Desviación típica ó Root Mean Square) de ambas fuentes de datos. 


\section{Modelo Utilizado}

Para efectuar las simulaciones numéricas, se utilizó el modelo numérico "Weather Research and Forecasting" (WRF), que es útil para pronóstico y sistema de asimilación de datos. El modelo se desarrolló como un esfuerzo de las principales instituciones de los EE.UU, con la participación de un gran número de científicos de diferentes universidades a nivel mundial [5]. WRF es conveniente para aplicaciones en un amplio espectro de casos, desde escalas de metros a los millares de kilómetros. Se puede utilizar para la investigación y la predicción numérica operacional de las condiciones atmosféricas, asimilación de datos e investigación de parametrizaciones físicas, "downscaling" dinámico y estadístico".

\section{Caraterísticas del modelo WRF}

El modelo WRF consta de varios módulos, donde el principal es el Avanced Research Weather (ARW). Las principales características de este Módulo son las siguientes [5]:

- Tiene un sistema de ecuaciones completamente comprensibles. Es conservativo para las variables escalares.

- Presión de aire seco. Opcionalmente, energía cinética turbulenta y muchos otros escalares.

- La coordenada vertical está representada en el sistema de coordenadas sigma que se ajusta a la topografía y presión hidrostática. La grilla horizontal es Arakawa C.

- Utiliza un esquema de Runge-Kutta de tercer orden con pequeño incremento del paso de tiempo, para modos de ondas acústicas y de gravedad.

- Discretización espacial: Usa esquemas del 2do al 6to orden, con opciones de advección en la coordenada horizontal y vertical.

- Rotación de la Tierra : Todos los términos de Coriolis incluidos.

\section{Métodos}

Se implementó el modelo atmosférico WRF, en El Centro de Modelización numérica del Instituto del Mar del Perú (IMARPE), utilizando "scripts"' desarrollados por investigadores de IRD (Francia). Se efectuaron simulaciones con este modelo para el año 2000, con una resolución espacial de $90 \mathrm{~km}$, con grilla anidada de $30 \mathrm{~km}$ de resolución sobre una parte del Océano Pacífico Suroriental, con fronteras, entre las latitudes $3^{\circ} \mathrm{N}-23^{\circ} \mathrm{S}$ y $90^{\circ} \mathrm{O}-$ $69^{\circ} \mathrm{O}$ (Figura 1 ), utilizando como condiciones iniciales y de frontera los datos de Reanálisis [6].
El pre-procesamiento de los datos de entrada al modelo WRF se debe realizar, para la conversión de los datos con formato NetCDF a formato binario y ubicar las variables a la grilla del modelo. Por otro lado, para el postprocesamiento de la información, para la conversión de formato NetCDF a binario y ASCII, se utiliza el software "Grid Análisis and Display System" (GrADS). También, se utiliza "Generic Mapping Tools" (GMT), Scripts en Bash, Cshell, Gnuplot, lenguajes Fortran, AWK entre otros, ya que con estas herramientas informáticas se puede procesar y visualizar datos de ciencias de la Tierra en diferentes formatos como GRIB, Binario, NetCDF, ASCII, etc. Para este análisis, se elaboraron mapas de promedios bimensuales de las componentes zonales y meridionales de velocidad superficial del viento, RMS-diff y correlación de las componentes de velocidad entre los datos de QuikSCAT y WRF.

\section{Resultados}

Para comparar la variabilidad de la circulación atmosférica modelada y observada, se utilizaron datos de viento para el periodo 2000 obtenidos de la base de datos internacionales QuikSCAT y datos de viento obtenidos de simulaciones mediante el modelo atmosférico WRF. EI ámbito geográfico en el que se realizó el estudio es una parte del Océano Pacífico suroriental, entre los paralelos $23^{\circ} \mathrm{S}-3^{\circ} \mathrm{N}$ y meridianos $90^{\circ} \mathrm{O}-69^{\circ} \mathrm{O}$ (Figura 1 ).

\section{Análisis de velocidades}

Los datos originales del modelo WRF, tienen salidas horarias. Estos datos fueron procesados para obtener promedios diarios, mensuales, bimensuales y estacionales. Los cálculos se realizaron para las componentes zonal $(u)$, meridional $(v)$ y para el vector resultante.

- Promedios Bimensuales de las Componentes de Velocidad : El procesamiento de datos de QuikSCAT, WRF y Reanálisis, para la componente zonal $u$, da como resultado estructuras similares para las tres fuentes de datos. Las principales discrepancias, entre los datos de QuikSCAT y de WRF se encuentran en las zonas cercanas a la costa, donde la diferencia entre ambas fuentes de datos oscila entre 1 a $2 \mathrm{~m} / \mathrm{s}$. Para las áreas alejadas de la costa, las coincidencias son mayores, a la vez que conforme la distancia a la costa aumenta, los valores absolutos de la componente $u$, también aumentan. La diferencia entre los productos presentados es significativa, sin embargo, WRF simula vientos meridionales con gradientes perpendiculares a la costa (cross-shore) reducidos en comparación con los Reanálisis, lo que es más realista. 
Para la componente meridional $v$, se encuentra que la distribución de velocidades se incrementa, hasta llegar a un máximo (5-7 m/s) y luego decrece al aumentar la distancia hacia la costa. En la Figura 2, se muestra las diferencias de RMS, y las correlaciones de los datos de QuikSCAT y WRF, donde se observa que las mayores correlaciones se presentan lejos de la línea de costa (mayores a 0.7 ), mientras que para regiones cercanas a la costa las correlaciones son menores a 0.4 .

- Promedios Bimensuales de la Magnitud de Velocidad En las Figuras 2 y 3 se muestra la magnitud de las velocidades horizontales promedio del viento para el período de estudio, con el fin de ilustrar las discrepancias entre los datos utilizados. Se observa que los datos reanalizados de NCEP no captan las estructuras finas del viento debido a la resolución espacial gruesa en que se encuentran $\left(2.5^{\circ}\right.$ de latitud por $2.5^{\circ}$ de longitud). No obstante la distribución de las velocidades dentro del área del estudio es similar en todos los casos, con la diferencia que los datos simulados mediante el modelo WRF están fuertemente influenciados por los valores de los forzantes utilizados. Pero la similitud con los datos observados de QuikSCAT son evidentes, como también se verá más adelante, donde se presenta los mapas de correlaciones entre los datos de QuikSCAT y los de WRF. Aquí, se observa que los valores de velocidad modelados y observados difieren principalmente en la zona ecuatorial y en menor grado en la franja costera. El modelo regional simula velocidades horizontales mayores en algunos puntos de la franja costera donde la inclinación de la línea costera aumenta (por ejemplo en $14^{\circ} S$ y $6^{\circ} S$ de latitud). Además, la velocidad simulada es más débil en las zonas donde el ángulo de la costa disminuye $\left(19^{\circ} \mathrm{S}\right.$ de la latitud). En las Figuras 2 (b), (e) y (h), se ilustra los datos de velocidad de viento, obtenidos mediante el modelo WRF promediados para los meses de Enero - Febrero, Marzo - Abril, y Mayo-Junio, respectivamente. Aquí, se puede observar que las velocidades horizontales modeladas promediadas bimensualmente (Figuras 2 (b), (e) y (h)), generalmente son mayores que las observadas ( $\mathrm{Fi}$ guras 2 (a), (d) y (g)), en las áreas alejadas de la costa. Esto se observa claramente en los núcleos de máximas velocidades que se representa en cada gráfico, donde el área ocupada por el viento superficial con velocidades mayores a $7.5 \mathrm{~m} / \mathrm{s}$ son de mayor extensión a la representada por los datos observados de QuikSCAT. Para los meses de Julio-Agosto, Setiembre-Octubre y Noviembre-Diciembre (Figuras 3 a-i), la distribución de velocidades generadas con el modelo WRF (Figuras $3 \mathrm{~b}$, e y $\mathrm{h}$ ) reproduce bien las velocidades observadas de QuikSCAT. Las velocidades obtenidas mediante Reanálisis (Figuras 3F, 3f, y 3), simulan velocidades mayores que los de los datos de WRF y QuikSCAT (Figuras 3a, 3 y $3 \mathrm{~g}$ ).

- RMS de las Componentes de Velocidad

Para estudiar la variabilidad de las componentes horizontales del viento, con respecto a su media, y de la magnitud resultante de las componentes horizontales de la velocidad del viento, se calculó el valor cuadrático medio (Root Mean Square: RMS), en cada punto de la grilla, sobre toda el área de estudio, utilizando las siguientes ecuaciones[7]:

$$
\begin{aligned}
R M S_{u} & =\sqrt{\frac{\sum_{i=1}^{N T}\left[u_{i}-\bar{u}\right]^{2}}{N T}} \\
R M S_{v} & =\sqrt{\frac{\sum_{i=1}^{N T}\left[v_{i}-\bar{v}\right]^{2}}{N T}} \\
R M S & =\sqrt{\frac{\sum_{i=1}^{N T}\left[\left|\mathbf{v}_{i}\right|-|\overline{\mathbf{v}}|\right]^{2}}{N T}}
\end{aligned}
$$

Donde: $u$ y $v$ son las componentes zonal y meridional del viento; $\bar{u}=\frac{\sum_{i=1}^{N T} u_{i}}{N T}$ y $\bar{v}=\frac{\sum_{i=1}^{N T} v_{i}}{N T}$ son las medias aritméticas de $u$ y $v ;\left|\mathbf{v}_{i}\right|$ es la magnitud horizontal del viento, para un instante de tiempo $i$ $;\left|\mathbf{v}_{\mathbf{i}}\right|=\sqrt{u_{i}{ }^{2}+v_{i}{ }^{2}}$ es la magnitud horizontal de la velocidad del viento [7]; $N T=366$ es el número de días correspondientes al año de simulación, durante el 2000. $R M S_{u}$ es la variabilidad de la componente $u$, que es ilustrada en las Figuras 4a, 40 y 4 f, correspondiente a los datos de QuikSCAT, WRF y Reanálisis, respectivamente; $|\overline{\mathbf{v}}|=\frac{\sum_{i=1}^{N T} \mid \mathbf{v}_{i}}{N T}$ es la media aritmética de la velocidad horizontal del viento.

En las figuras mencionadas, se observa que las $R M S_{u}$, de los datos de WRF son muy parecidos a los de QuikSCAT, desde los $23^{\circ} \mathrm{S}-15^{\circ} \mathrm{S}$ de latitud aproximadamente, con valores superiores a $1.5 \mathrm{~m} / \mathrm{s}$. Para el área desde los $15^{\circ} \mathrm{S}-3^{\circ} \mathrm{N}$ de latitud, los valores de RMS de WRF presentan menores valores que los de QuikSCAT del orden de $0.25 \mathrm{~m} / \mathrm{s}$. Los datos de Reanálisis presentan estructuras similares a los de WRF y QuikSCAT, aunque existen diferencias en los valores numéricos.

La componente $R M S_{v}$ es la variabilidad de la componente horizontal $v$. Estos datos son ilustrados en las Figuras $4 \mathrm{~d}, 4 \mathrm{k}$ y $4 \mathrm{f}$. Se observa que tiene una mayor variabilidad que la componente $u$. De la misma forma que para los datos de $R M S_{u}$, los valores de WRF son muy parecidos a los de QuikSCAT en la parte sur, del área de estudio y presentan diferencias en la parte central y norte de la misma. Los datos de 
$R M S_{v}$ de Reanálisis, muestran mayores diferencias, en comparación con las otras fuentes de datos, ya que presenta una franja de alta variabilidad paralela a la costa, lo cual no es representada en los datos observados de QuikSCAT. Esta componente es la que tiene predominancia en el cálculo de RMS total de la velocidad horizontal del viento (Figuras, 4]).

\section{Correlación de Velocidades}

Para estudiar las similitudes entre los datos de QuikSCAT y de WRF se calculó las correlaciones respectivas mediante las expresiones [7]:

$$
\begin{aligned}
r_{u} & =\frac{\frac{\sum_{i=1}^{N T}\left(u_{Q(i)}-\bar{u}_{Q}\right)\left(u_{W(i)}-\bar{u}_{W}\right)}{N T}}{\sigma_{u Q} \sigma_{u W}} \\
r_{v} & =\frac{\frac{\sum_{i=1}^{N T}\left(v_{Q(i)}-\bar{v}_{Q}\right)\left(v_{W(i)}-\bar{v}_{W}\right)}{N T}}{\sigma_{v Q} \sigma_{v W}}
\end{aligned}
$$

Donde: El subíndice $u$, indica la componente zonal y $v$, indica la componente meridional. Además $r_{u} \mathrm{y}$ $r_{v}$, son las correlaciones de las componentes horizontales de la velocidad del viento, en las direcciones $u$ (zonal) y $v$ (meridional); $u_{Q(i)}$ y $v_{Q(i)}$ son las componentes horizontales $u$ y $v$ de los datos de velocidad del viento de QuikSCAT; $u_{W(i)}, v_{W(i)}$, son las componentes horizontales, zonal $u$ y meridional $v$ de los datos de WRF; $\bar{u}_{Q}$ y $\bar{u}_{W}$ son los promedios anuales de la componente zonal $u$ de la velocidad del viento de los datos de QuikSCAT y WRF, para cada punto de grilla sobre el área de estudio; similarmente $\bar{v}_{Q}$ y $\bar{v}_{W}$ representan los promedios de la componente meridional $v$ de la velocidad del viento de ambas fuentes de datos.

Además, $\sigma_{u Q}, \sigma_{u W}$ son las desviaciones estándar de las conponentes zonales de QuikSCAT y WRF; $\sigma_{v Q}$, $\sigma_{v W}$ son las desviaciones estándar de las componentes meridionales de QuikSCAT y WRF; $N T=366$ es el número de días del año 2000.

En la Figura 6r, se muestra las correlaciones de la componente zonal $u$, entre los datos de QuikSCAT y WRF. Aquí, se observa que las correlaciones en la parte costera en el sur y norte del Perú están entre los $0.2-0.4$, mientras que en la zona central (alrededor de Paracas) las correlaciones llegan hasta 0.7. Para las zonas alejadas de la costas los valores de correlación aumentan significativamente, llegando hasta 0.9 . Si se trazan perfiles perpendiculares a la costa, los valores de correlación aumentan cuando se incrementa la distancia hacia la costa.

En la Figura 6d se muestra los valores de correlación para la componente meridional $v$, entre los datos de QuikSCAT y WRF. Las correlaciones cerca de la costa presentan valores mayores a 0.4 para el Norte y Sur del Perú y mayores a 0.5 para la zona central. Los valores de correlación aumentan, conforme la distancia hacia la costa crece, tomando valores entre $0.6-0.9$.

- RMS de Magnitud de la Velocidad En la Figura 4 se presenta los RMS de los datos de QuikSCAT, Reanálisis y WRF. Los gráficos tienen representaciones similares para todos los casos, pero la principal diferencia se debe a que los datos de WRF presentan mayores áreas con baja variabilidad, en comparación con los datos de QuikSCAT.

- Asimetría de la Magnitud de Velocidad del Viento Para estudiar la simetría de la distribución de las velocidades horizontales del viento, se calculó la asimetría ("skewness") de los datos, utilizando la expresión [7]:

$$
\text { asimetria }=\frac{\sum_{i=1}^{N}\left(x_{i}-\bar{x}\right)^{3}}{N \sigma^{3}}
$$

Donde: $x_{i}$ son los valores que toma la variable en cada instante de tiempo; $\bar{x}$ es la media aritmética; $\mathrm{N}$ es el número de días del año y $\sigma=\left(\sum_{i=1}^{N}\left(x_{i}-\bar{x}\right)^{2} /(N-1)\right)^{1 / 2}$ es la raíz cuadrada de la varianza: En las Figuras $5 \mathrm{a}, 5 \mathrm{~b}$ y 5 ; se muestra la asimetría de la componente $v_{x}$ de la velocidad del viento, para los datos de QuikSCAT, WRF y Reanálisis. Para esta componente, los datos para la parte sur (hacia el sur de los $18^{\circ} \mathrm{S}$ de latitud) tienen valores positivos de asimetría altos en la mayor parte del tiempo. Aproximadamente a los $15^{\circ} \mathrm{S}$ de latitud, cambia de signo, indicando que para la parte central, predominan los valores por debajo de la media. Hacia la parte norte, desde los $18^{\circ} \mathrm{S}$ de latitud, los valores de asimetría tienden a tomar valores negativos, disminuyendo con la latitud, pero se presentan algunos núcleos con valores positivos alrededor de los $9^{\circ} S$ de latitud.

En las Figuras 54, 5e y 5f se representa los valores de asimetría para los datos QuikSCAT, WRF y Reanálisis. Para este caso los valores son negativos en casi toda el área de estudio, indicando, que para la componente $v_{y}$ la mayoría de datos tienen valores por debajo de la media, pero se presentan algunos núcleos de valores positivos, alrededor de los $14^{\circ} \mathrm{S}$ de latitud.

\section{Patrón del Esfuerzo de Viento}

Para obtener el campo de esfuerzos o flujo de momentum, a partir de los valores de las componentes de las velocidades superficiales, se utilizó la ecuación:

$$
\vec{\tau}=\left(\tau_{u}, \tau_{v}\right)=\rho C_{D}|\mathbf{v}|(u, v)
$$


Donde: $\tau$ es el vector esfuerzo horizontal de viento, $\tau_{u}$ es la componente zonal del esfuerzo de viento, $\tau_{v}$ es la componente meridional de la velocidad del viento, $\rho$ es la densidad; $C_{D}$ es el coeficiente de arrastre, y $|\mathbf{v}|$ es la magnitud de la velocidad horizontal del viento [8], [9], [10].

Los gráficos de esfuerzo de viento calculados mediante la ecuación 4, muestran gran similitud con los datos de velocidades promedio, ya que para calcular los valores del esfuerzo, el principal parámetro es la velocidad del viento.

\section{Análisis de Esfuerzos}

A partir de las componentes horizontales $\tau_{u}$ y $\tau_{v}$ de velocidad del viento, se calculó los esfuerzos diarios, mensuales, bimensuales y estacionales.

\section{Promedios Bimensuales del Esfuerzo de Viento}

En las Figuras $7 \mathrm{p}-\mathrm{i}$ y $8 \mathrm{p}-\mathrm{i}$, se muestran los gráficos de los datos de esfuerzo, obtenidos de las velocidades de las tres fuentes analizadas: Todos los gráficos mencionados, son similares a las representaciones de velocidades mostradas en las Figuras 2 y 3 .

\section{RMS de Esfuerzo del Viento}

Para hallar las componentes y magnitudes de los valores de RMS, se utilizó fórmulas similares a las ecuaciones 2 En las Figuras 9a, 9p y 9k se muestran los valores de RMS de la componente zonal $\left(\tau_{u}\right)$, del esfuerzo de viento. En ellas, se muestra que la variabilidad de esta componente en la zona costera es mayor en los datos de QuikSCAT y Reanálisis que para los del modelo WRF. La componente meridional $\left(\tau_{v}\right)$ y la de magnitud (Figuras $9 \mathrm{~d}, \mathrm{e}, \mathrm{f}, \mathrm{g}$, $\mathrm{h}$ e i) presentan mayores similitudes que el caso anterior entre los datos de QuikSCAT y WRF, pero los valores de Reanálisis presentan diferencias significativas con respecto a las otras fuentes de datos.

\section{Asimetría del Esfuerzo de Viento}

En las Figuras 10 a-f, se muestra los gráficos de la asimetría del esfuerzo del viento, para los datos de QuikSCAT, WRF y Reanálisis, respectivamente. Para los tres casos, la componente zonal $u$ (Figuras 10, 10p y 10 f), presenta valores negativos para las áreas adyacentes a la costa y valores positivos sobre la parte oceánica. Esto indica que valores por debajo de la media aritmética, predominan en el periodo de estudio. Para el caso de la componente meridional $v$ (Figuras $10 \mathrm{~d}, 10 \mathrm{p}$ y $10 \mathrm{f}$ ), todas presentan valores negativos en la mayor parte del área de estudio, presentando valores positivos sólo en áreas adyacentes a la ciudad de Paracas, en los datos de QuicSCAT y WRF. Esto indica que para la componente meridional del viento predominan los datos de velocidad con valores bajos, con relación al promedio.

\section{Rotacional del Esfuerzo de Viento}

El rotacional del esfuerzo de viento es una componente de fuerza importante en el transporte total verticalmente integrado de la circulación media del océano. Las regiones de corrientes de frontera del este, a la que pertenece el área de estudio, son caracterizadas por el rotacional anticiclónico del esfuerzo del viento, en las regiones costa afuera y tienden a ser ciclónicas cerca de ella, como se puede observar en los mapas del rotacional medio del esfuerzo de viento de las Figuras $11 \mathrm{a}-\mathrm{i}$ y $12 \mathrm{a}-\mathrm{i}$. Los valores del rotacional de las componentes horizontales del esfuerzo del viento, han sido calculados mediante la expresión [11], [12], [13]:

$$
\nabla \times \vec{\tau}=\beta \frac{\partial \vec{\psi}}{\partial x}=\frac{\partial \tau_{y}}{\partial x} \hat{i}-\frac{\partial \tau_{x}}{\partial y} \hat{j}
$$

Donde: $\beta$ es la derivada respecto a $y$ del parámetro de Coriolis; $\psi$ es la función de líneas de corriente del transporte de masa; $x$ e $y$ son las coordenadas en las direcciones este y norte, respectivamente; $\tau_{x}$ y $\tau_{y}$ son las componentes $x$ e $y$ del esfuerzo de viento, sobre la superficie del océano. Las descripciones realizadas del lugar de estudio, indican que el rotacional es ciclónico a lo largo de la costa,y tiene máximos valores en las vecindades de San Juan y al norte de Chimbote, y el rotacional es anticiclónico cerca a la costa de Tacna [14. Esto es reproducido por el modelo WRF, pero no se observa en los datos de QuikSCAT y de Reanálysis. Observamos diferencias sistemáticas en estos últimos campos del rotacional, no exhiben diferencias espaciales de la estructura cerca del área costera. Particularmente, productos de gran escala no demuestran una mayor variabilidad cerca de la costa, y pueden ser caracterizados por un rotacional negativo con amplitud pequeña, respecto al rotacional del esfuerzo de viento simulado por WRF, que muestra valores mayores (en valor absoluto) en el rotacional, que los de QuikSCAT. Esto tendrá, evidentemente, un impacto en la respuesta oceánica, que favorecerá los procesos que ocurran cerca de la costa.

\section{Conclusiones}

La finalidad de este estudio, fue la implementación del modelo WRF, para efectuar simulaciones sobre el área de estudio. Para ello se realizó un análisis comparativo entre los datos observados de QuikSCAT y WRF, donde se comprobó que los datos del modelo, reproducen adecuadamente los patrones de circulación atmosférica superficial, es por ello, que se puede establecer las siguientes conclusiones:

1. Se obtuvo buenos resultados simulando la circulación atmosférica utilizando el modelo WRF, para el año 2000, con una resolución espacial de $90 \mathrm{~km}$, con grilla anidada de $30 \mathrm{~km}$. Esta simulación se realizó para una parte del Océano Pacífico Suroriental, 
comprendida entre las latitudes $3^{\circ} \mathrm{N}-23^{\circ} \mathrm{S}$ y longitudes $90^{\circ} \mathrm{O}-69^{\circ} \mathrm{O}$.

2. Los análisis de variabilidad (RMS) y correlación, muestran el alto grado de similitud entre los datos de viento superficial simulados con el modelo WRF y de QuikSCAT, por lo que se concluye que los datos obtenidos mediante simulación numérica, con el modelo atmosférico WRF, reproduce adecuadamente la circulación atmosférica sobre el área de estudio.

3. Los datos, obtenidos mediante simulación con el modelo de circulación atmosférica WRF, son útiles para reemplazar a los forzantes atmosféricos observados, en las simulaciones con modelos de circulación oceánica, y en particular con el modelo ROMS, Estudio que será presentado en un artículo posterior.

\section{Agradecimientos}

Se agradece al Instituto del Mar del Perú por permitir el uso de sus instalaciones y de su centro de cómputo para la realización de este trabajo y a EI Instituto de Investigación para el Desarrollo (IRDFrancia), por el apoyo brindado.

\section{Referencias}

[1] H. Hendon, Brand Lienmann and John Glick; Oceanic Kelvin Waves and Madden-Julian Oscillations, J. Atmos. Sci., 55, 88-101 (1998)

[2] C. Zhang and Jonathan Gottshalck; SST Anomalies of ENSO and the Madden Julian Oscillation, in the Equatorial Pacific, J. Climate, 15, 2429-2445 (2002).

[3] A. Enriquez and C. Friehe; Effects of wind Stress and Wind Stress Curl Variability on Coastal UpweIling, J. of Phys. Oceanog., 25, 1651-1671 (1995).

[4] X. Capet, P. Marchesiello, and J.C., McWilliams; Upwelling response to coastal wind profiles, Geophys. Res. Lett., 31 (2004)

[5] W. Skamarock, J. Kemp, J. Dudhia, D. Gill, D.Barker, M. Duda, Xiang-Yu Huang, Wei Wang, and Jordan G. Powers; A Description of Advanced Research WRF Versión 3, Mesoscale and Microscale Meteorology División, National Center for Atmospheric Research, Boulder, Colorado, USA. NCAR/TN-475+STR, NCAR TECNICAL NOTE, June 2008, 125 pp, (2008).

[6] E. Kalnay, M. Kanamitsu, R. Kistler, W. Collins, D. Deaven, L. Gandin, M. Iredell, S. Saha, G. White, J. Woollen, Y. Zhu, A. Leetmaa, R. Reynolds, M. Chelliah, W. Ebisuzaki, W. Higgins, J. Janowiak, K. C. Mo, C. Ropelewski, and J. Wang; The NCEP/NCAR 40 Year Reanalysis Project, Bull. Amer. Meteor. Soc. 77, 437-471 (1996).
[7] Spiegel Murray; Estadística, teoría y aplicaciones. Mc GRAW-HILL, 357 pp (1974).

[8] D. Fissel, Pond, and M. Miyake; Computation of surface fluxes from climatological and sinoptic data. Mon. Wea. Rev. 105, 26-37 (1976).

[9] Andrew Bunker; Computations of surface energy flux and Annual Air-sea interactions cycles of the north atlantic ocean, Mon. Wea. Rev. 104, 11221140 (1976).

[10] Steven Esbensen and Richard Reynolds; Estimating monthly averaged air - sea transfers of heat and momentum using the bulk aerodynamic method, J. Phys. Oceanog., 11, 457,506 (1981).

[11] S. Hellerman; Computations of wind stress fields over the Atlantic Ocean, Mon. Wea. Rev. 'emph93, 607-623 (1965).

[12] S. Hellerman; An updated estimate od the wind stress of the world ocean, Mon. Wea. Rev. 95, 239244 (1967).

[13] D. Harrison; On climatological monthy mean wind stress and wind stress curl fields over the world ocean, J. Climate, 2, 57-71 (1989).

[14] A. Bakun and C. Nelson; The seasonal cycle of wind stress curl in sub-tropical eastern boundary current regions. J. of Phys. Oceanog. 21, 1815-1834 (1990). 


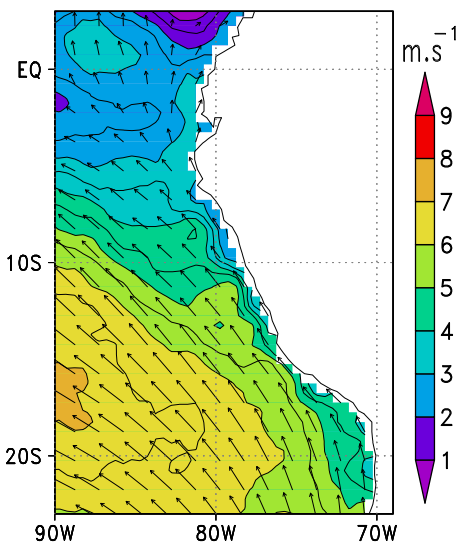

(a) Enero-Febrero; QuikSCAT

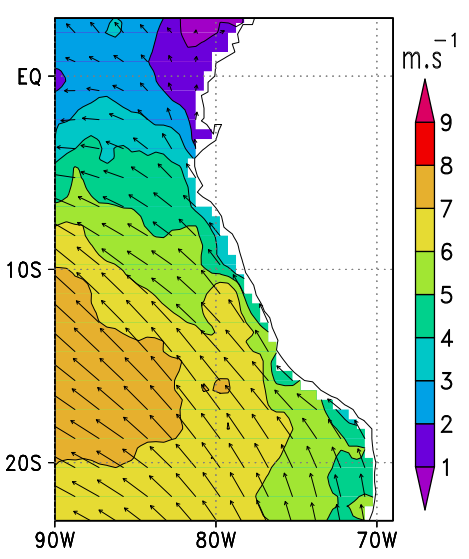

(d) Marzo-Abril; QuikSCAT

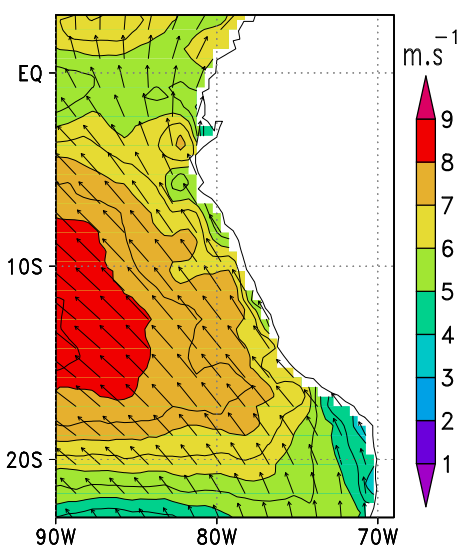

(g) Mayo-Junio; QuikSCAT

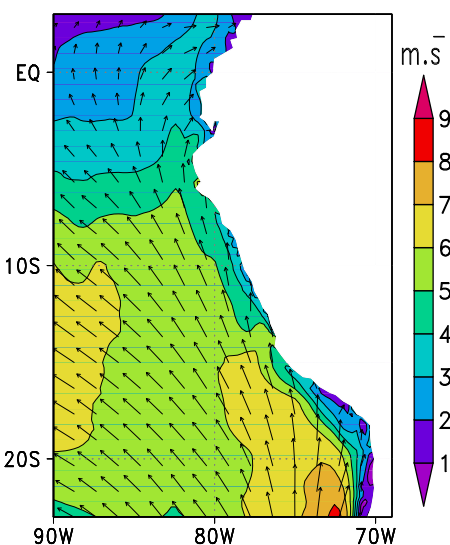

(b) Enero-Febrero; WRF

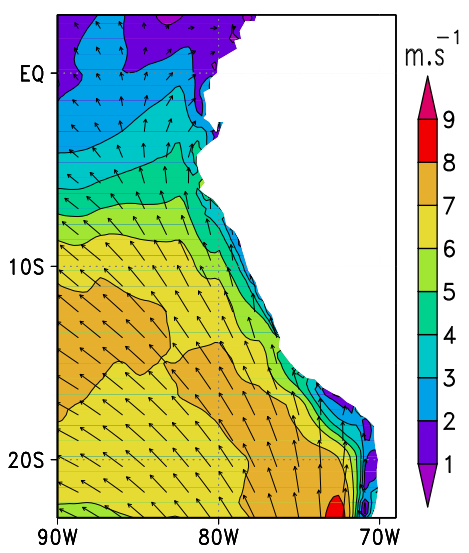

(e) Marzo-Abril; WRF

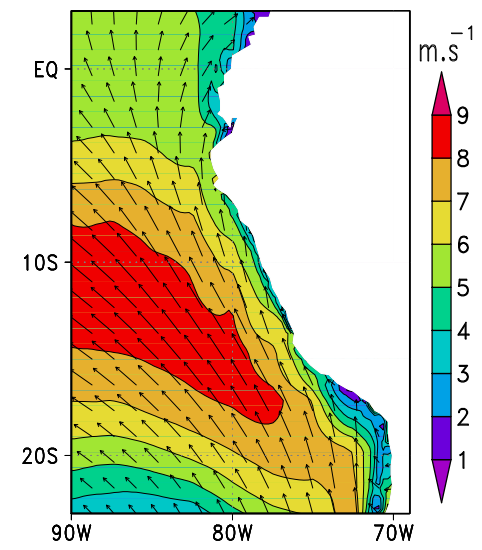

(h) Mayo-Junio; WRF

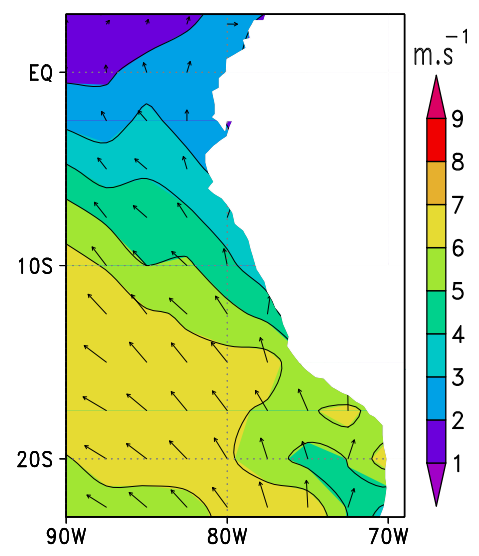

(c) Enero-Febrero; Reanálisis

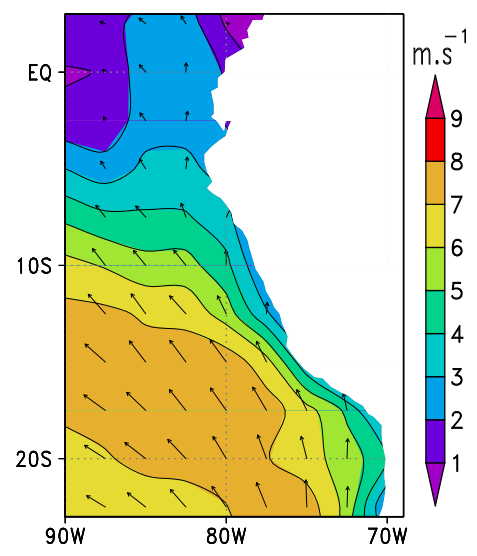

(f) Marzo-Abril; Reanálisis

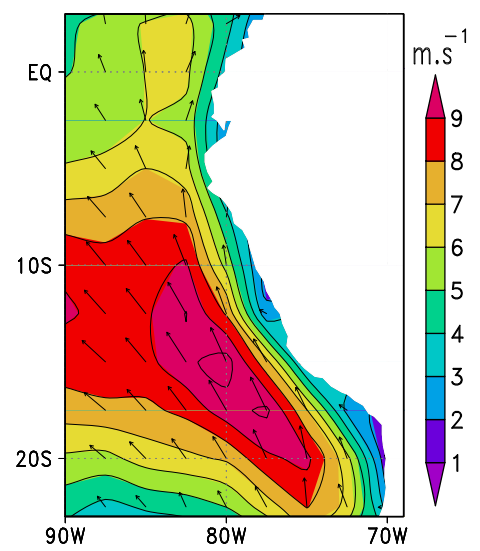

(i) Mayo-Junio; Reanálisis

Figura 2: Promedio de la velocidad horizontal del viento para los meses de Enero - Febrero (fila superior), Marzo - Abril (fila intermedia), y Mayo - Junio (fila inferior), para el año 2000. En las figuras se presentan los datos de: QuikSCAT a, d, g; WRF b, e, h ; Reanálisis y c, f, i. Los colores indican la magnitud de la velocidad la que varía desde violeta $(1 \mathrm{~m} / \mathrm{s})$ hasta rojo $(9 \mathrm{~m} / \mathrm{s})$, con intervalos de $1 \mathrm{~m} / \mathrm{s}$. Las tres fuentes de datos presentan estructuras similares, y en todas la velocidad del viento aumenta al transcurrir los meses. 


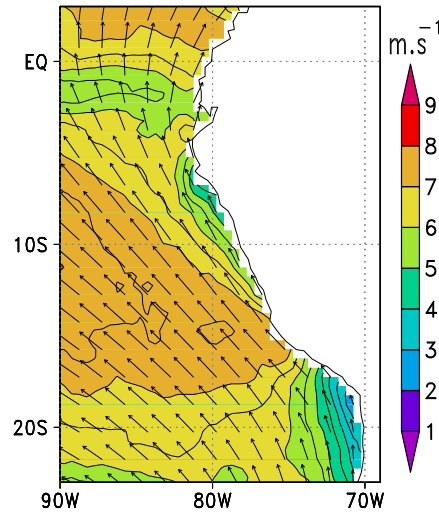

(a) Julio-Agosto; QuikSCAT

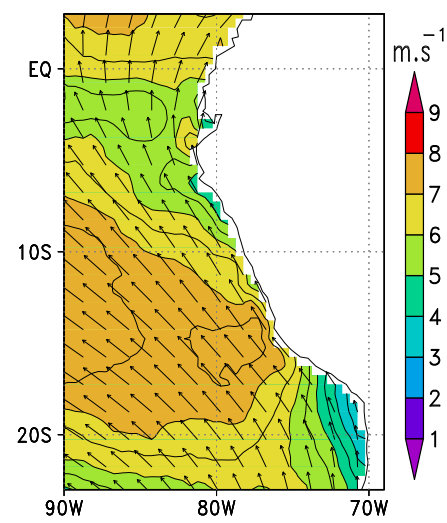

(d) Setiembre-Octubre; QuikSCAT

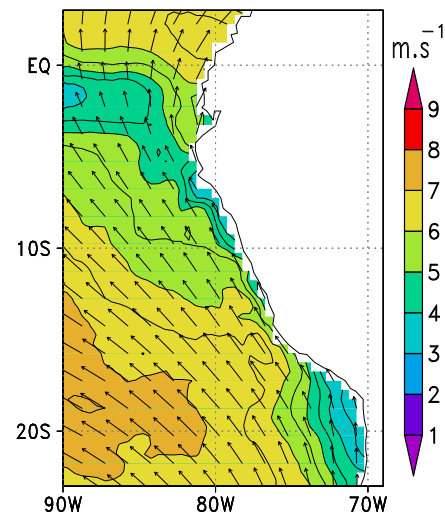

(g) Noviembre-Diciembre; QuikSCAT

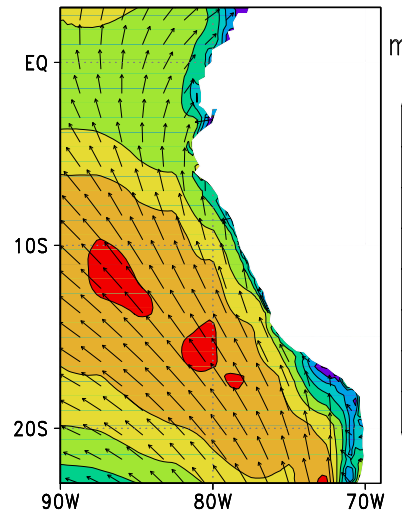

(b) Julio-Agosto; WRF

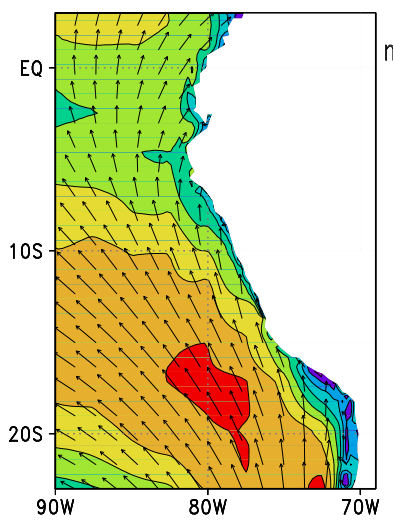

(e) Setiembre-Octubre; WRF

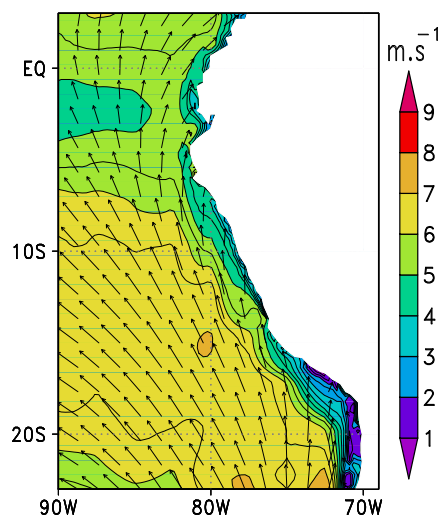

(h) Noviembre-Diciembre; WRF

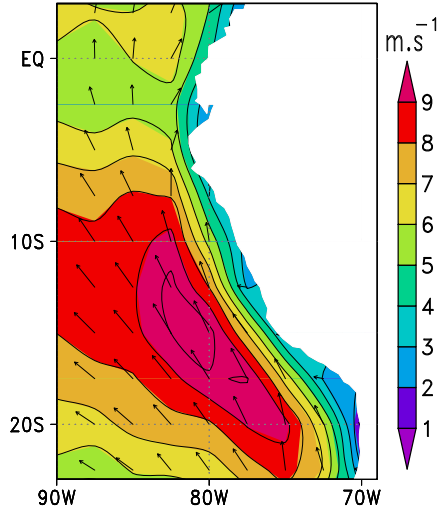

(c) Julio-Agosto; Reanálisis

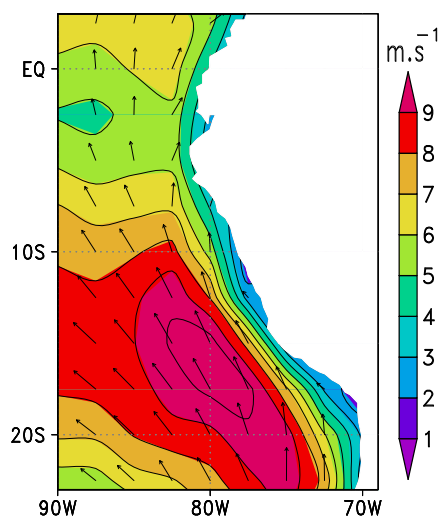

(f) Setiembre-Octubre; Reanálisis

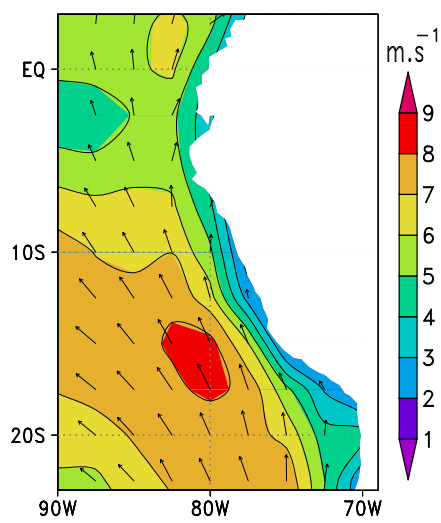

(i) Noviembre-Diciembre; Reanálisis

Figura 3: Promedio de la velocidad horizontal del viento para los meses de Julio - Agosto (fila superior), Setiembre Octubre (fila intermedia), y Noviembre - Diciembre (fila inferior), para el año 2000. En las figuras se presentan los datos de: QuikSCAT a, d, g (columna izquierda); WRF b, e, h (columna central);Reanálisis c, f, i de (columna derecha). Los colores indican la magnitud de la velocidad, la que y varía desde violeta $(1 \mathrm{~m} / \mathrm{s})$ hasta rojo $(9 \mathrm{~m} / \mathrm{s})$, con intervalos de $1 \mathrm{~m} / \mathrm{s}$. Las tres fuentes de datos presentan estructuras similares, y en todas, la velocidad del viento alcanza su maxima intensidad para los meses de Julio - Agosto, para luego en los meses siguientes. 


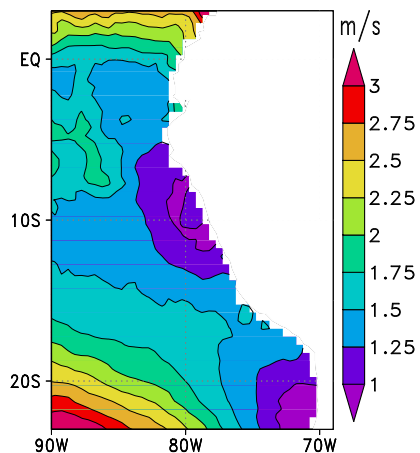

(a) RMS de la componente " $u$ "; QuikS CAT

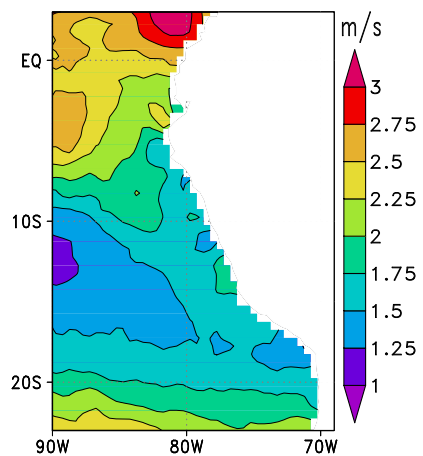

(d) RMS de la componente " $v$ "; QuikS CAT

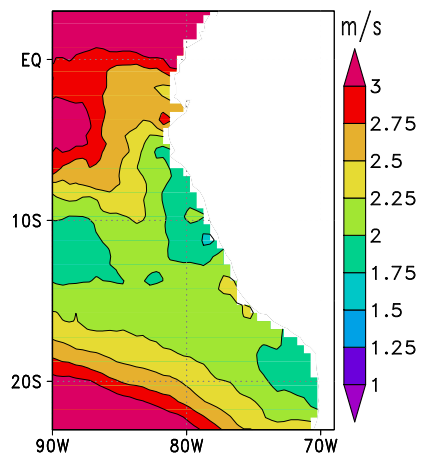

(g) RMS de la magnitud del viento; QuikSCAT
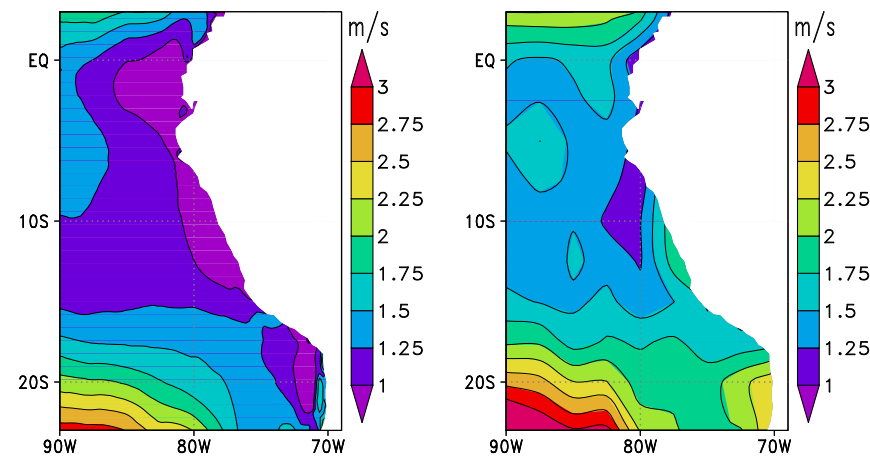

(b) RMS de la componente " $u$ "; WRF lisis
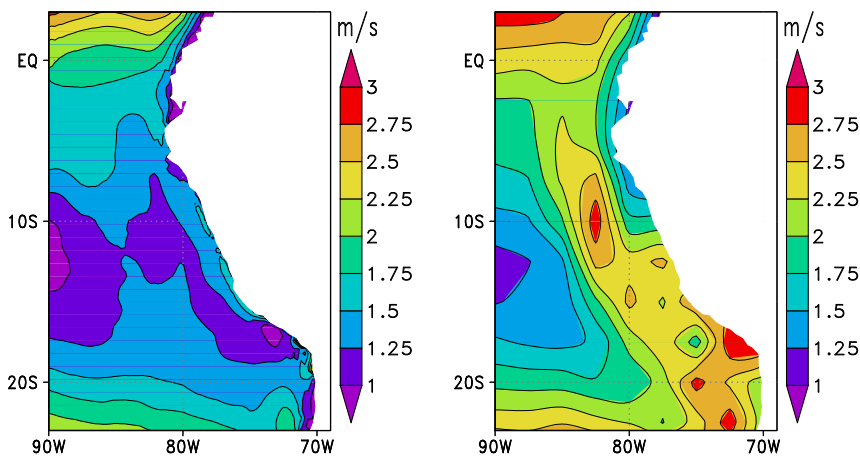

(e) RMS de la componente " $v$ "; WRF lisis
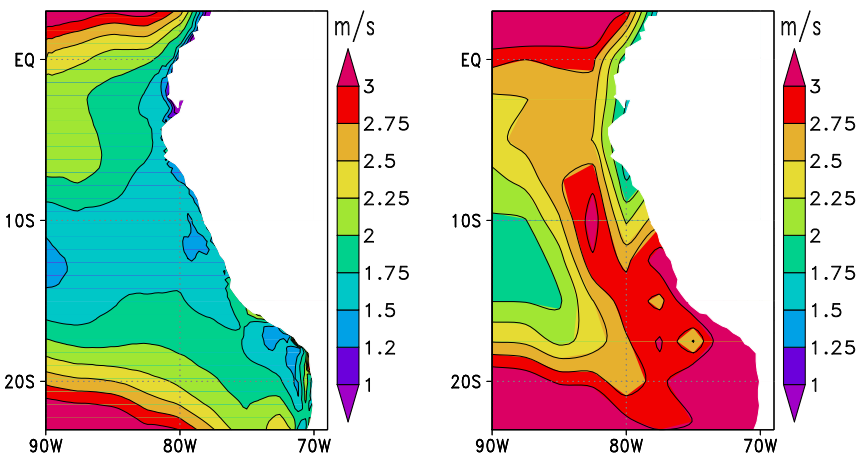

(h) RMS de la magnitud del viento; (i) RMS de la magnitud del viento WRF

Reanálisis

Figura 4: RMS (variabilidad) de viento horizontal, año 2000, calculada con la ecuación 2 (a), (d) y (g) datos de QuikSCAT. (b), (e) y (h) datos de WRF. (c), (f) e (i) datos de Reanálisis. Los colores varían desde violeta $(1 \mathrm{~m} / \mathrm{s})$, hasta rojo $(3 \mathrm{~m} / \mathrm{s})$, con intervalos de $0.25 \mathrm{~m} / \mathrm{s}$. Los valores de QuikSCAT presentan mayor variabilidad que los datos de WRF y Reanálisis. 


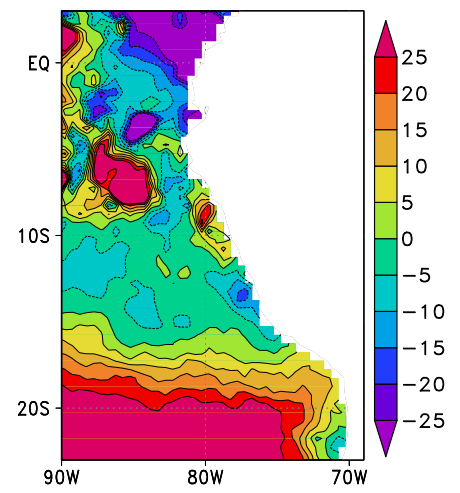

(a) Asimetría de la componente $u$, QuikSCAT

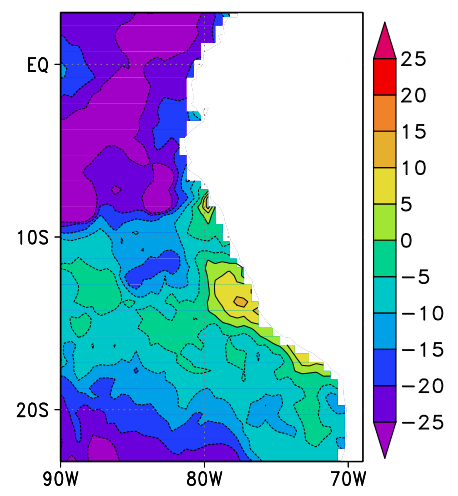

(d) Asimetría de la componente $v$, QuikSCAT

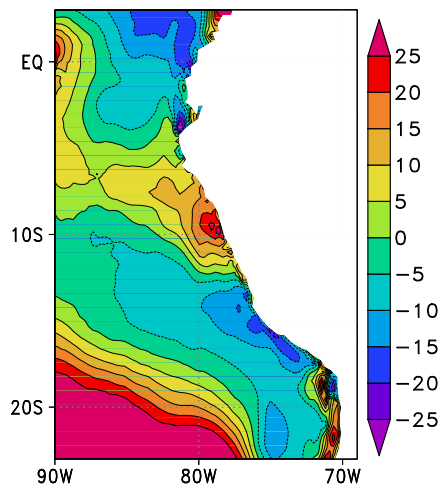

(b) Asimetría de la componente $u$,WRF

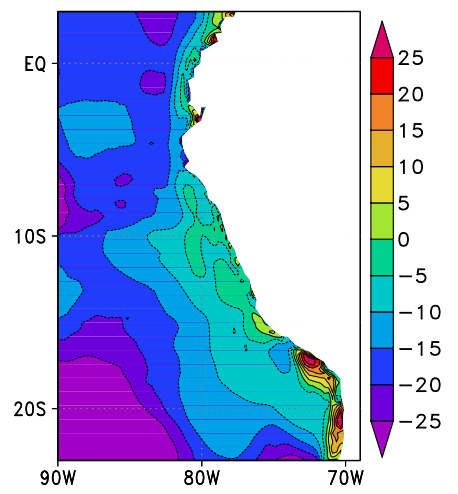

(e) Asimetría de la componente $v$ WRF

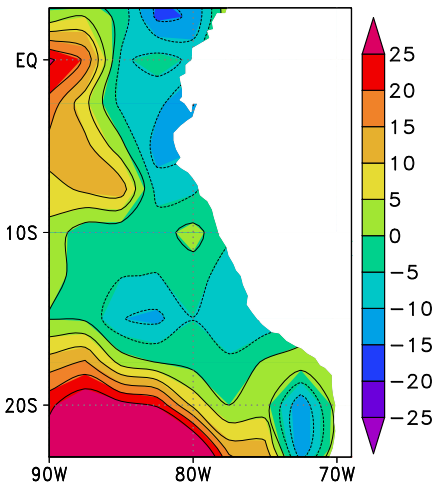

(c) Asimetría de la componente $u$, Reanálisis

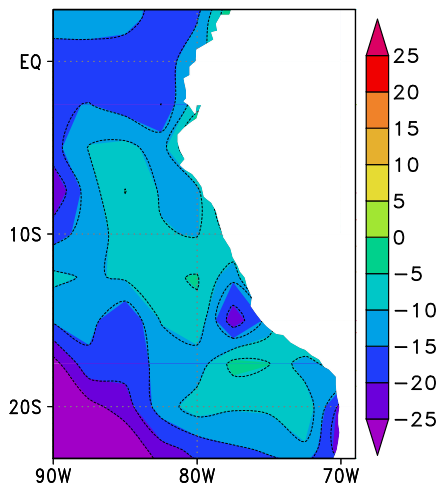

lisis

Figura 5: Asimetría (skewness) de la velocidad del viento, año 2000. (a) y (d) datos de QuikSCAT. (b) y (e) datos de WRF. (c) y (f) datos de Reanálisis. Los colores varían desde violeta (-25) hasta rojo (25), con intervalos de 5 unidades. Para la componente $u$, en la parte sur (debajo de los $18^{\circ} \mathrm{S}$ de latitud, predominan los valores sobre la media, mientras que para el resto del área de estudio (hacia el norte de los $18^{\circ} \mathrm{S}$ de latitud, predominan los valores por debajo de la media. Para la componente $v$, predominan valores por debajo de la media en casi toda el area de estudio, con ecepción de la zona alrededor de la ciudad de Paracas. 


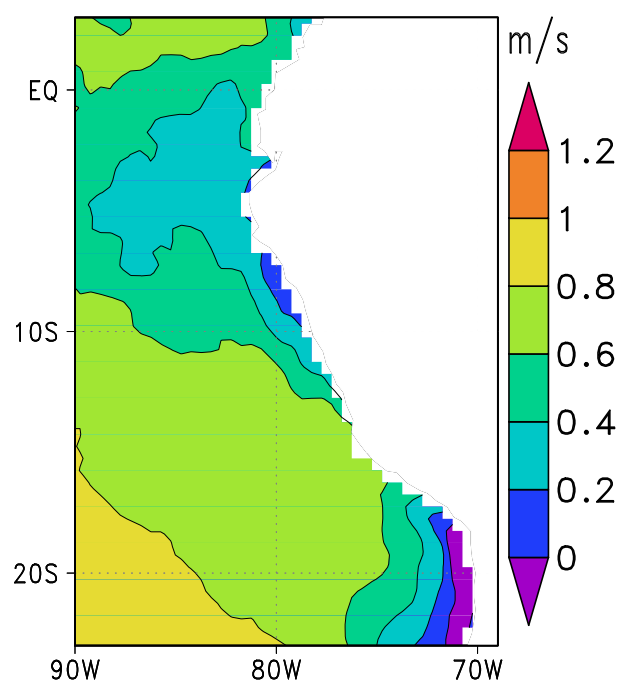

(a) RMS-diff, componente $u$, de los datos de WRF y (b) QuikSCAT

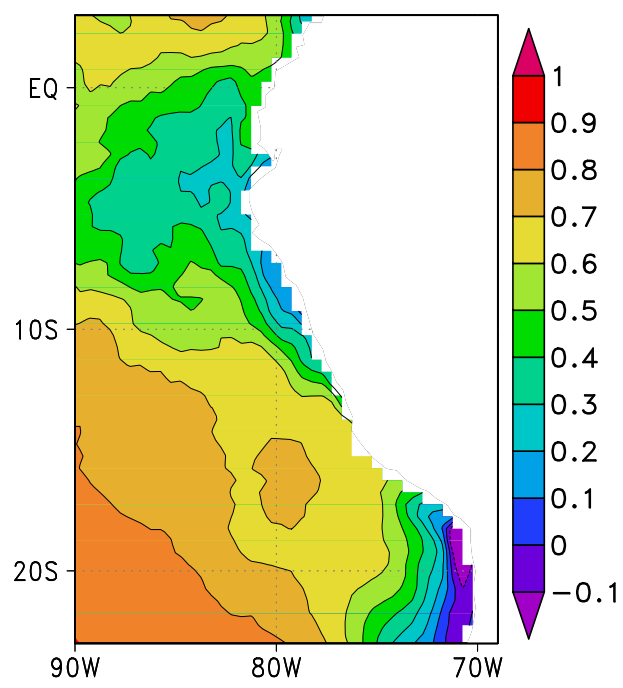

(c) Correlación de la componente $u$, de los datos de WRF (d) Correlación de la componente $v$, de los datos de WRF y QuikSCAT.

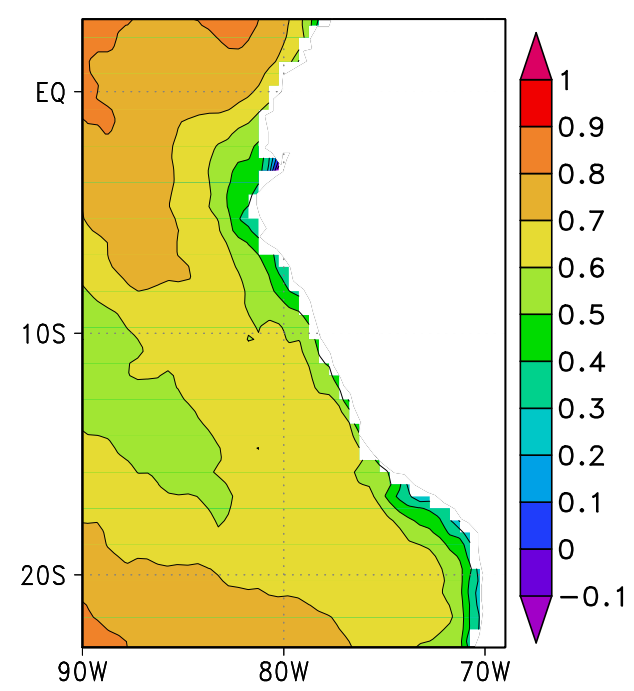

y QuikSCAT.

Figura 6: Las Figuras (a) y (b) muestran las RMS-diff de las componentes $u$ y $v$ entre los datos de WRF y QuikSCAT. Los colores varían desde azul $(0 \mathrm{~m} / \mathrm{s})$, hasta naranja $(1.2 \mathrm{~m} / \mathrm{s})$, con intervalos de $0.5 \mathrm{~m} / \mathrm{s}$. En las Figuras (c) y (d), se muestran los gráficos de correlación entre las componentes de velocidad. Los colores varían desde violeta $(-0.1)$, hasta rojo (1), con intervalos de 0.1 . Los valores negativos de correlación indican que al aumentar los valores de la velocidad de WRF, las velocidades de QuikSCAT tienden a disminuir, o viceversa. 


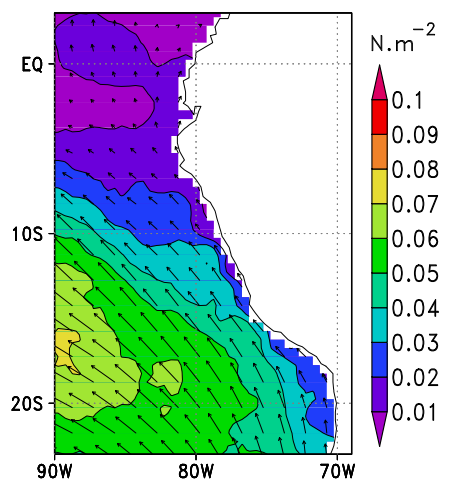

(a) Enero-Febrero; QuikSCAT

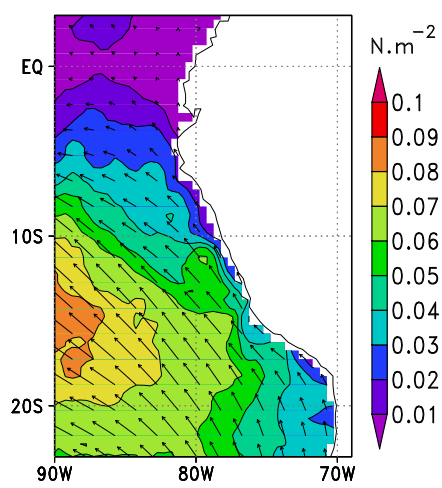

(d) Marzo-Abril; QuikSCAT

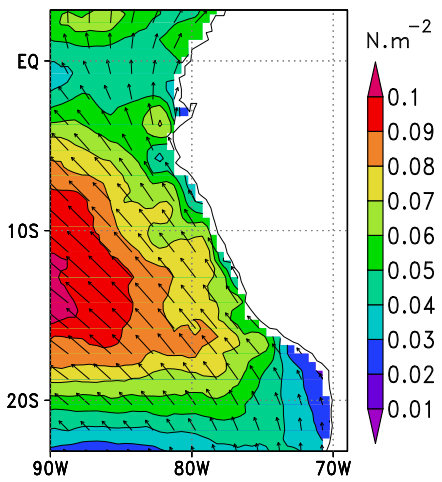

(g) Mayo-Junio; QuikSCAT

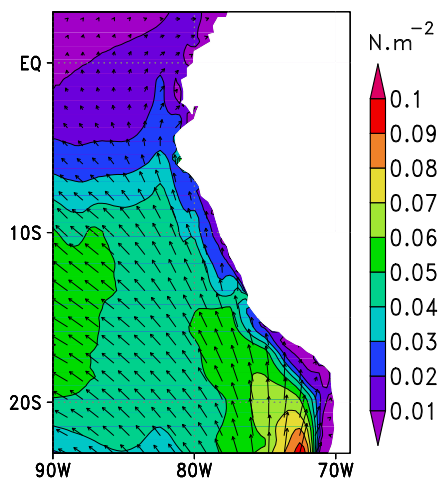

(b) Enero-Febrero; WRF

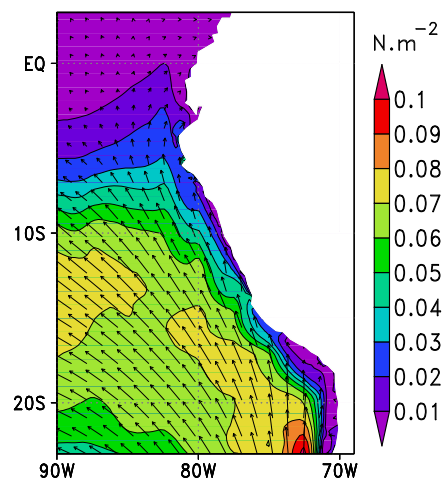

(e) Marzo-Abril; WRF

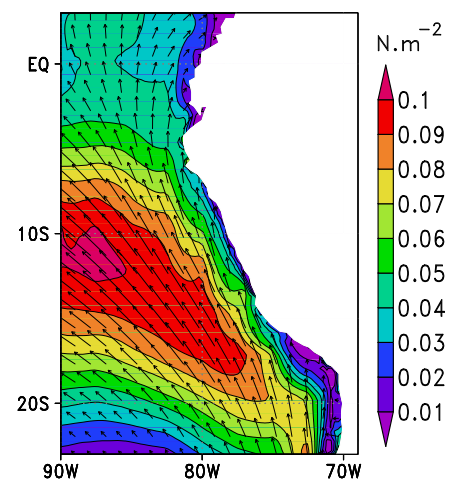

(h) Mayo-Junio; WRF

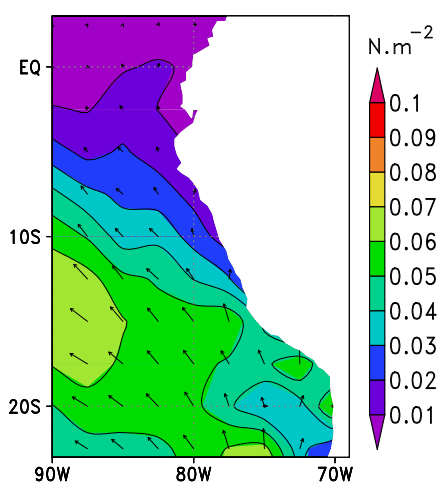

(c) Enero-Febrero; Reanálisis

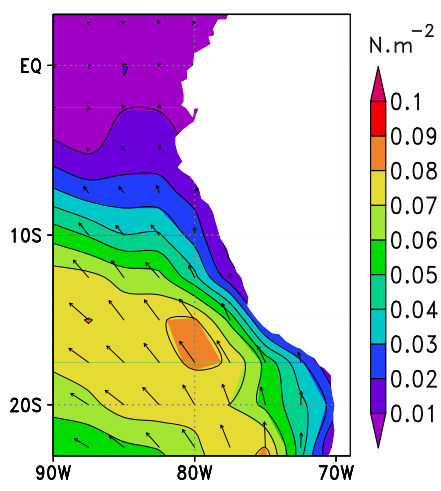

(f) Marzo-Abril; Reanálisis

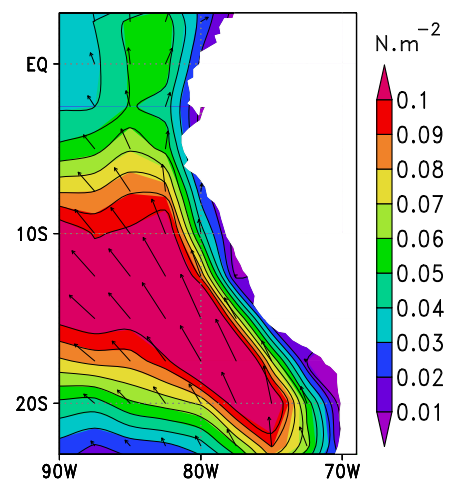

(i) Mayo-Junio; Reanálisis

Figura 7: Promedios del esfuerzo de viento, para los meses Enero - Febrero, Marzo - Abril, y Mayo - Junio del año 2000. (a), (d) y (g) datos de QuikSCAT (columna izquierda). (b), (e) y (h) datos de WRF (columna central) y (c), (f) e (i) datos de Reanálisis (columna derecha). Los colores indican la magnitud del esfuerzo, que varía desde violeta $\left(0.01 \mathrm{~N} . \mathrm{m}^{-1}\right)$ hasta rojo $\left(0.1 \mathrm{~N} . \mathrm{m}^{-1}\right)$, con intervalos de $0.01 \mathrm{~N} . \mathrm{m}^{-1}$. Los valores de esfuerzo tienen un núcleo máximo aproximadamente a los $15^{\circ} \mathrm{S}$ de latitud y $88^{\circ} \mathrm{O}$ de longitud, y aumentan al transcurrir los meses. Las flechas representan los vectores del esfuerzo de viento. 


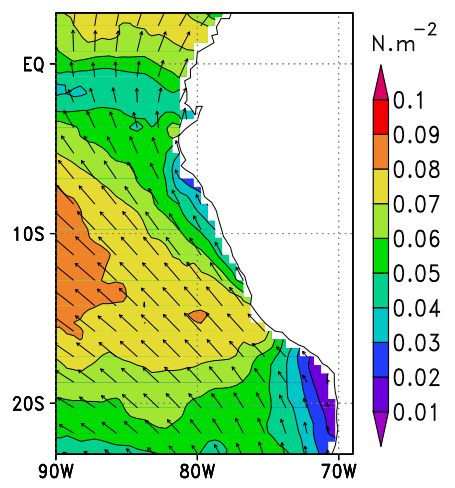

(a) Julio-Agosto; QuikSCAT

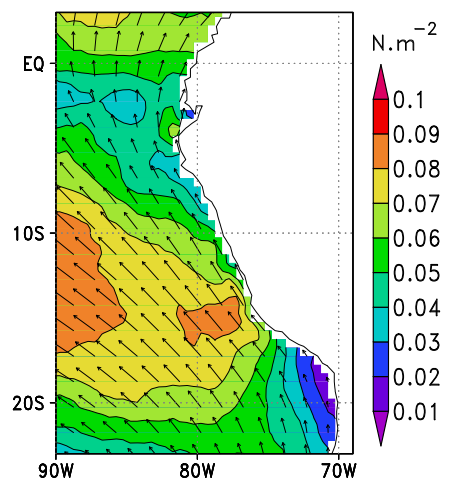

(d) Setiembre-Octubre; QuikSCAT

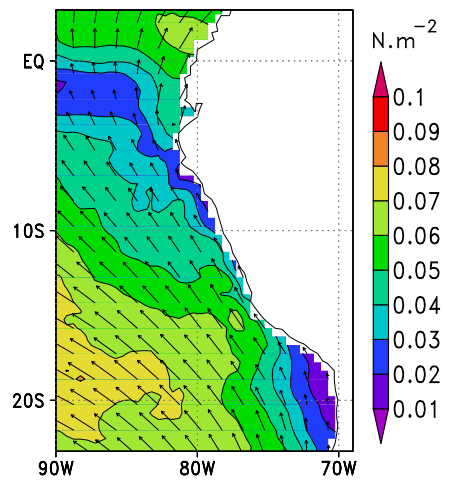

(g) Noviembre-Diciembre; QuikSCAT

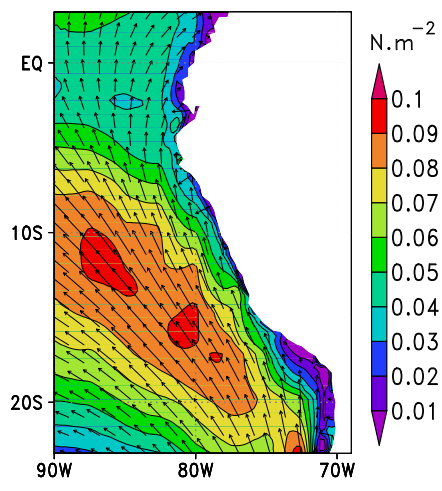

(b) Julio-Agosto; WRF

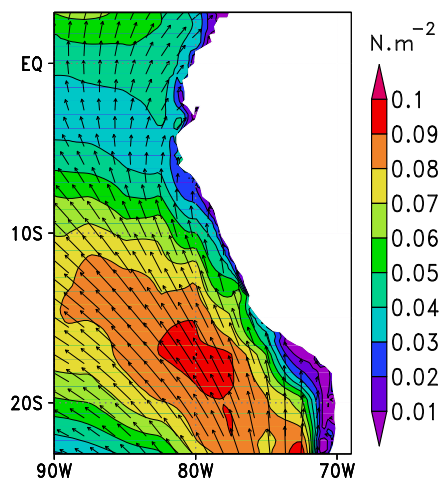

(e) Setiembre-Octubre; WRF

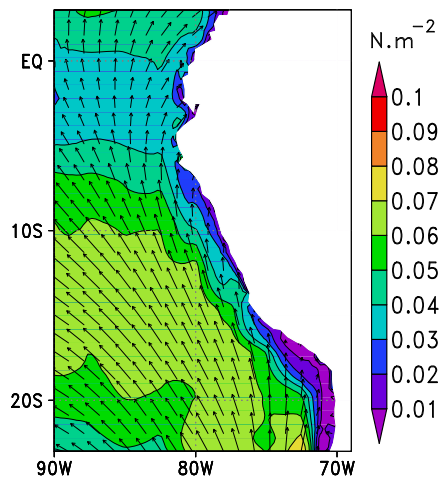

(h) Noviembre-Diciembre; WRF

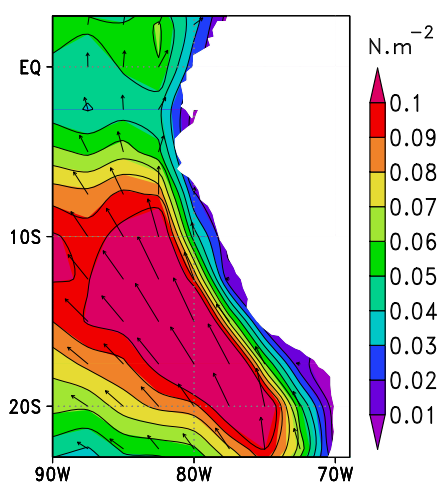

(c) Julio-Agosto; Reanálisis

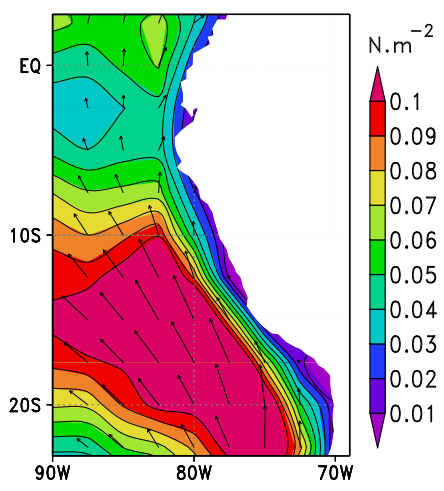

(f) Setiembre-Octubre; Reanálisis

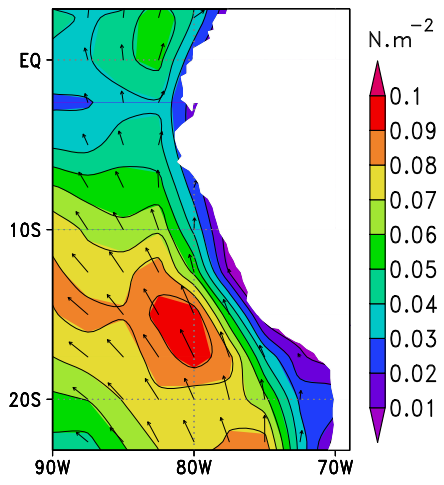

(i) Noviembre-Diciembre; Reanálisis

Figura 8: Promedios del esfuerzo de viento, para los meses Julio -Agosto, Setiembre - Octubre, y Noviembre - Diciembre del año 2000. Los gráficos representan los datos de QuikSCAT (columna izquierda), modelos WRF (columna central) y Reanálisis (columna derecha). Los colores indican la magnitud del esfuerzo, la que varía desde violeta $\left(0.01 N . \mathrm{m}^{-1}\right)$ hasta rojo $\left(0.1 \mathrm{~N} . \mathrm{m}^{-1}\right)$, con intervalos de $0.01 \mathrm{~N} . \mathrm{m}^{-1}$. Los valores de esfuerzo tienen su máximo valor entre Julio -Agosto y luego disminuyen progresivamente hasta los meses de Noviembre - Diciembre. Las flechas representan los vectores del esfuerzo de viento. 


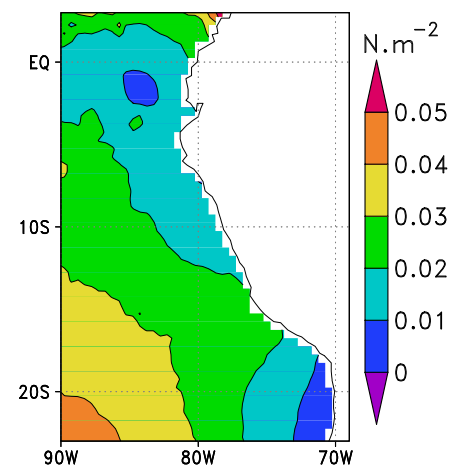

(a) Componente $\tau_{u}$ de RMS; QuikSCAT

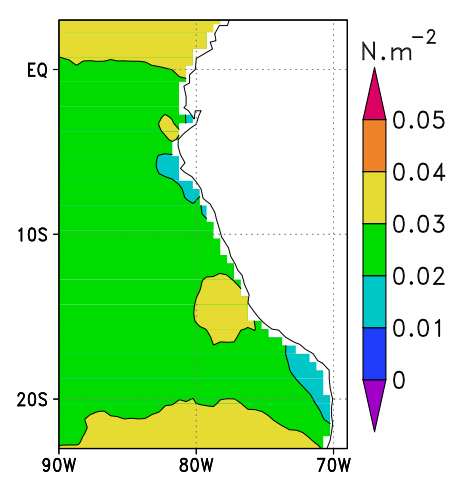

(d) Componente $\tau_{v}$ de RMS; QuikSCAT

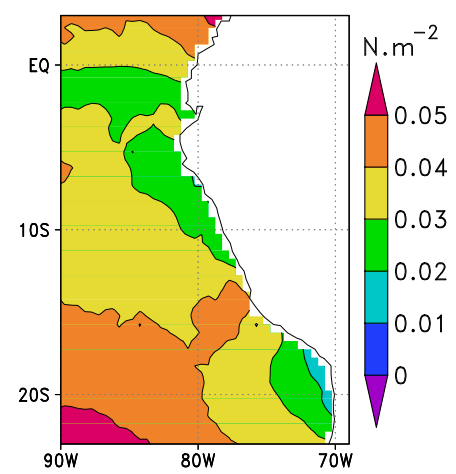

(g) RMS de la magnitud de $\tau$; QuikSCAT

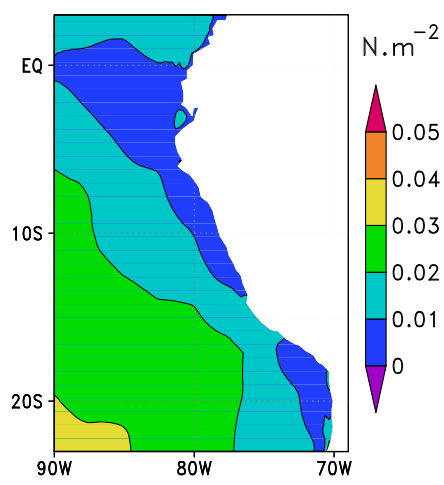

(b) Componente $\tau_{u}$ de RMS; WRF

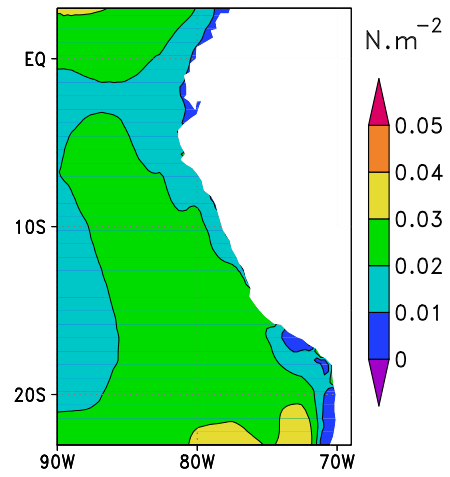

(e) Componente $\tau_{u}$ de RMS; WRF

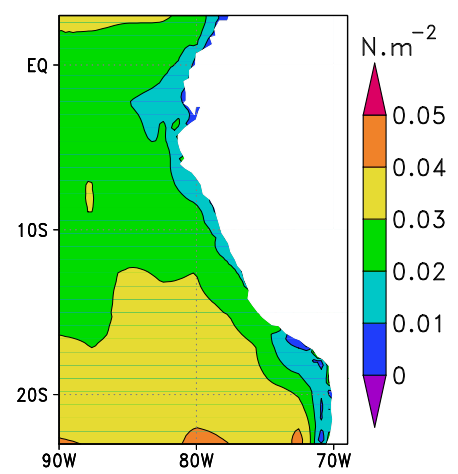

(h) RMS de la magnitud de $\tau$; WRF

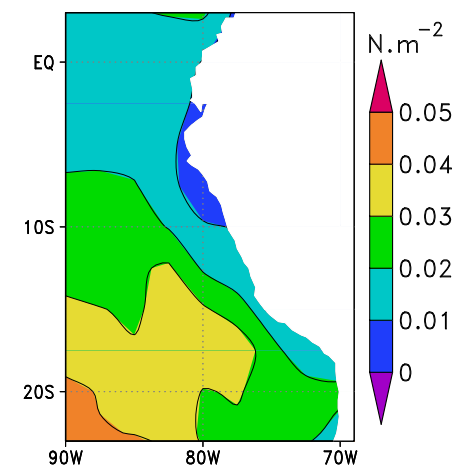

(c) Componente $\tau_{u}$ de RMS; Reanálisis

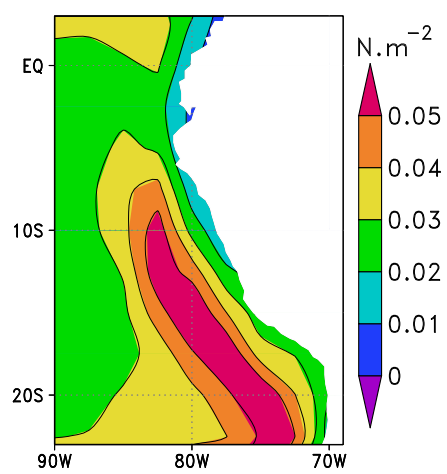

(f) Componente $\tau_{v}$ de RMS; Reanálisis

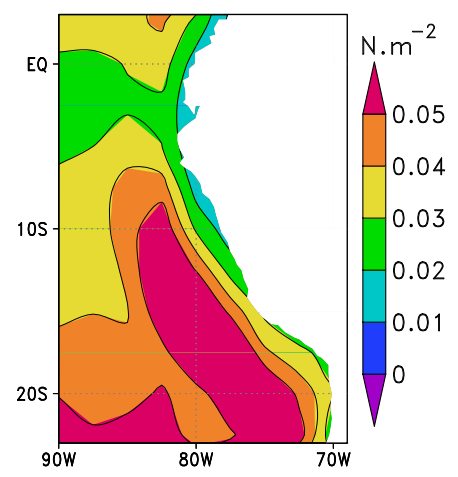

(i) RMS de la magnitud de $\tau$; Reanálisis

Figura 9: Los gráficos muestran los valores de RMS del esfuerzo de las componentes horizontales y de la magnitud de la velocidad del viento, para el año 2000. (a),(d) y (g) datos de QuikSCAT; (b), (e) y (h) datos de WRF; (c), (f) e (i) datos de Reanálisis. Los colores indican los valores de RMS del esfuerzo, que varía desde violeta $\left(0.00 \mathrm{~N} . \mathrm{m}^{-2}\right)$ hasta rojo $\left(0.05 \mathrm{~N} . \mathrm{m}^{-2}\right)$, con intervalos de $0.01 \mathrm{~N} . \mathrm{m}^{-2}$. Los valores de RMS de esfuerzo de QuikSCAT, presenta mayores valores que los de WRF en todos los casos. 


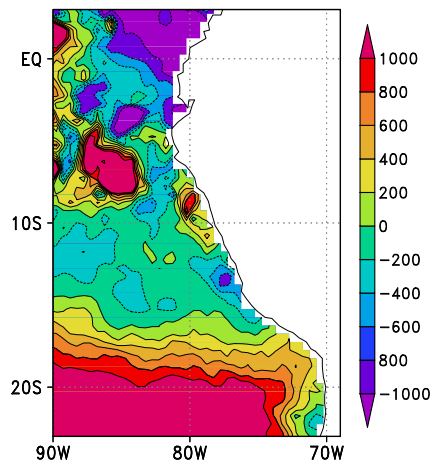

(a) componente zonal $\tau_{u}$ de Asimetría; QuikSCAT

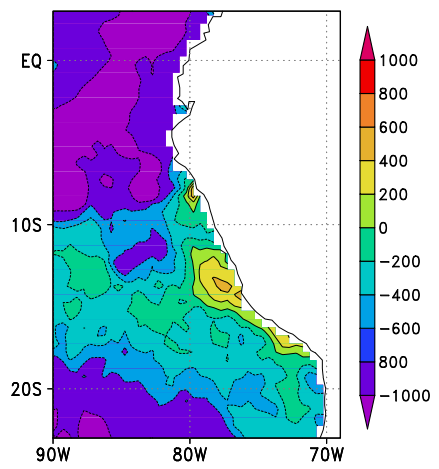

(d) Componente meridional $\tau_{v}$ de Asimetría; QuikSCAT

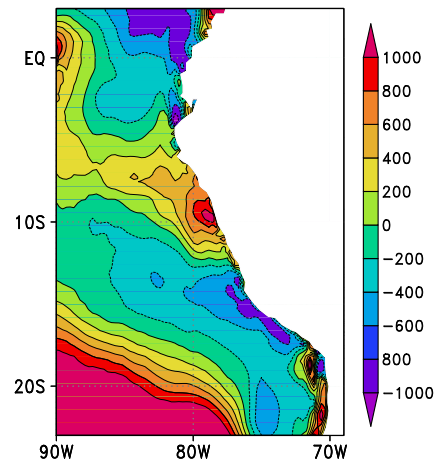

(b) componente zonal $\tau_{u}$ de Asimetría; WRF

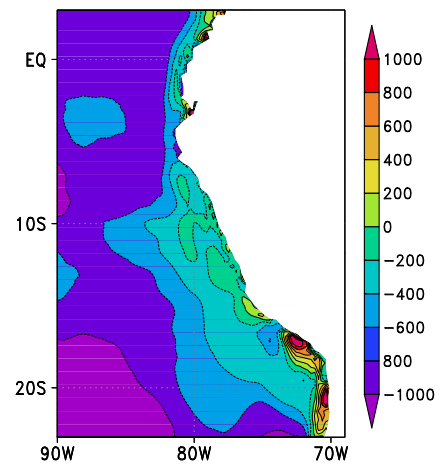

(e) componente meridional $\tau_{v}$ de Asimetría; WRF

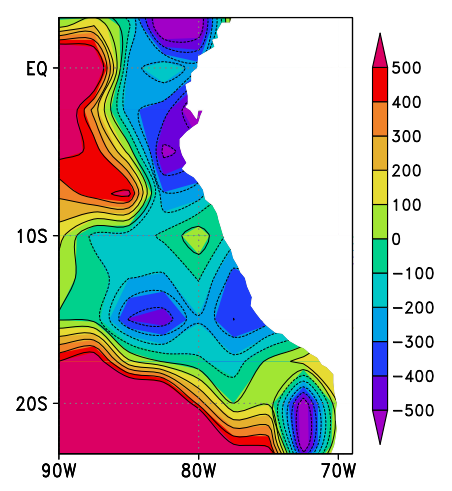

(c) componente zonal $\tau_{u}$ de Asimetría; Reanálisis

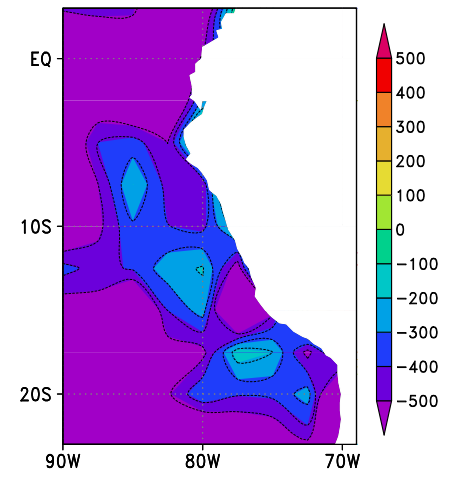

(f) componente meridional $\tau_{v}$ de Asimetría; Reanálisis

Figura 10: Asimetría de las componentes horizontales del esfuerzo de viento, para el año 2000; (a), (d) datos de QuikSCAT; (b), (e) datos de WRF; (c), (f) datos de Reanálisis. Los colores indican valores de asimetía, que varía desde violeta (-1000 unidades) hasta rojo (1000) unidades, con intervalos de 200 unidades. Para el caso de las figuras de Reanálisis, se ha representado una barra de colores diferente, por que la diferencia de sus valores, con las otras fuentes de datos es significativa. Los valores positivos de Asimetría indican que predominan los valores sobre la media, mientras que los valores negativos indican que predominan valores por debajo de la media. 


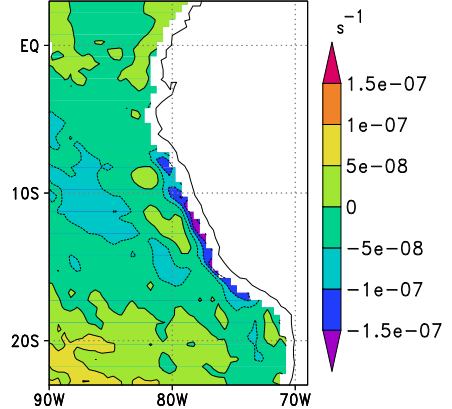

(a) Enero-Febrero; QuikSCAT

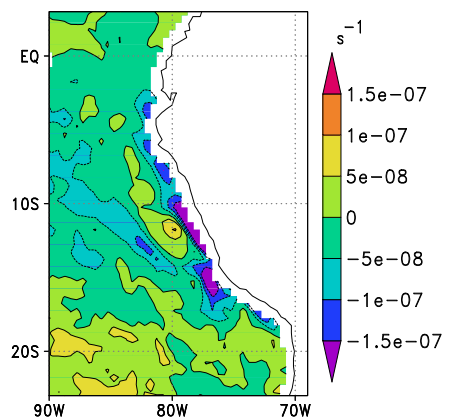

(d) Marzo-Abril; QuikSCAT

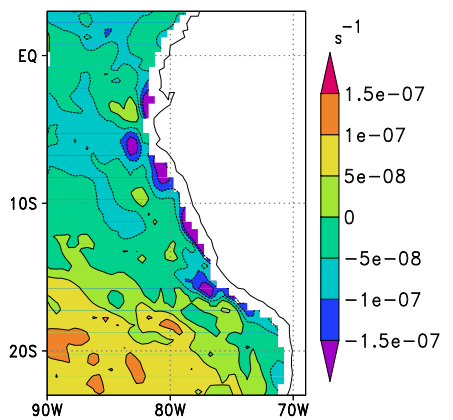

(g) Mayo-Junio; QuikSCAT

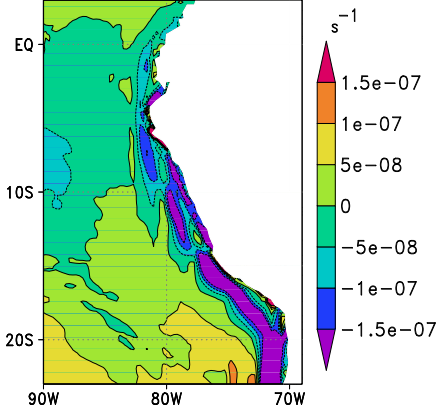

(b) Enero-Febrero; WRF

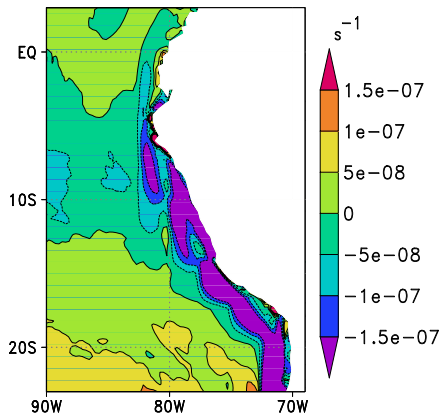

(e) Marzo-Abril; WRF

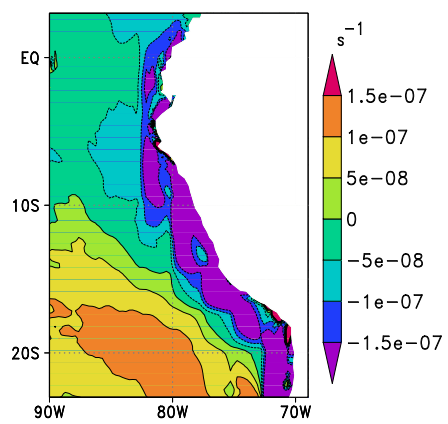

(h) Mayo-Junio; WRF

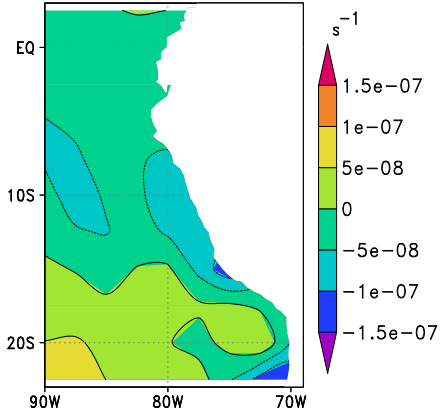

(c) Enero-Febrero; Reanálisis

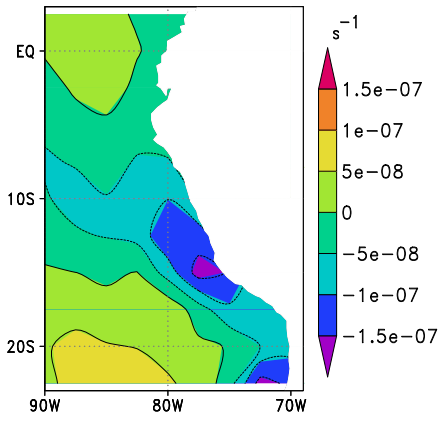

(f) Marzo-Abril; Reanálisis

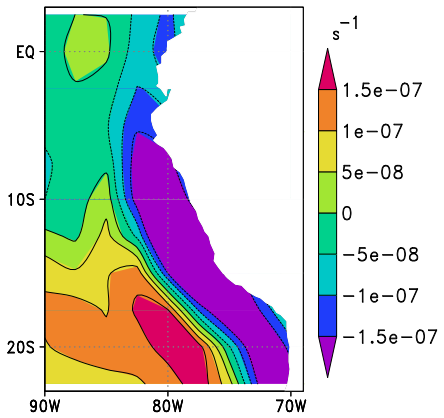

(i) Mayo-Junio; Reanálisis

Figura 11: Promedios de rotacional de esfuerzo de viento, para los meses Enero - Febrero, Marzo - Abril, y Mayo Junio del año 2000. (a), (d), (g) datos de QuikSCAT; (b), (e), (h) datos de WRF; (c), (f), (i) datos de Reanálisis. Los colores indican la magnitud del rotacional, la que varía desde violeta $\left(-1.5 \times 10^{-7} s^{-1}\right)$ hasta rojo $\left(1.5 \times 10^{-7} s^{-1}\right)$, con intervalos de $-1.5 \times 10^{-7} s^{-1}$. Los valores positivos indican rotaciones anticiclónicas (sentido antihorario) y los negativos indican rotaciones clónicas (en sentido de las agujas del reloj). El comportamiento del rotacional juega un papel muy importante para la generación de afloramiento de aguas sub-superficiales. 


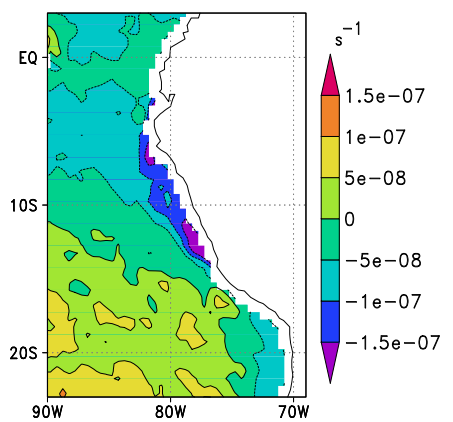

(a) Julio-Agosto; QuikSCAT

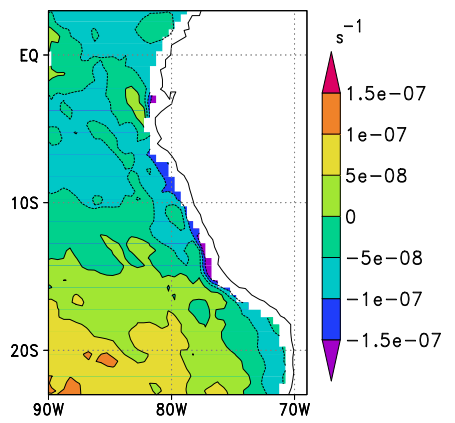

(d) Setiembre-Octubre; QuikSCAT

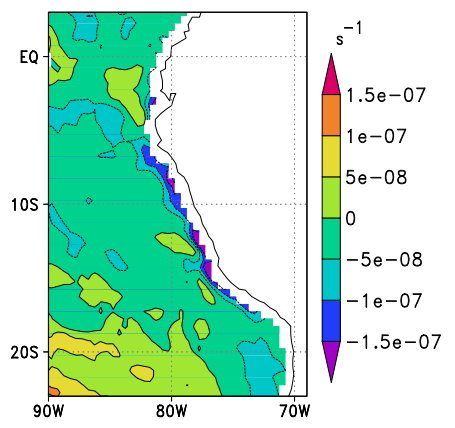

(g) Noviembre-Diciembre; QuikSCAT

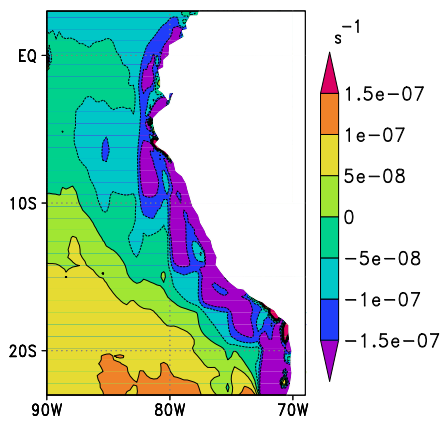

(b) Julio-Agosto; WRF

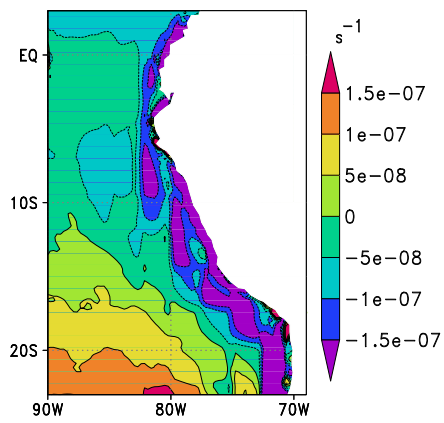

(e) Setiembre-Octubre; WRF

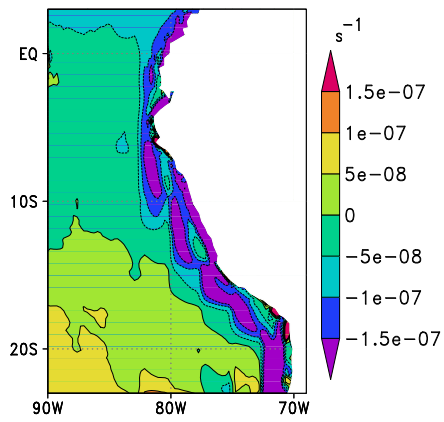

(h) Noviembre-Diciembre; WRF

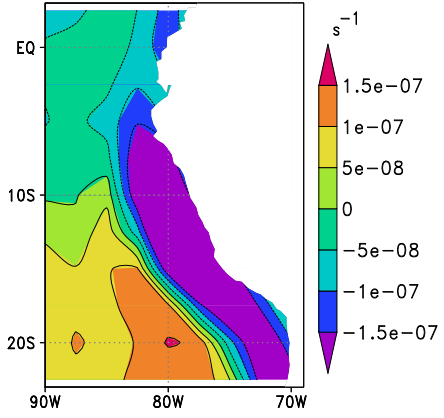

(c) Julio-Agosto; Reanálisis

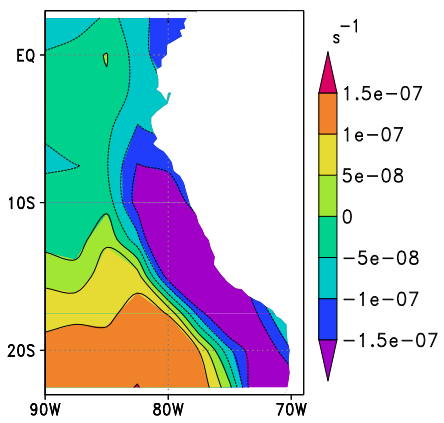

(f) Setiembre-Octubre; Reanálisis

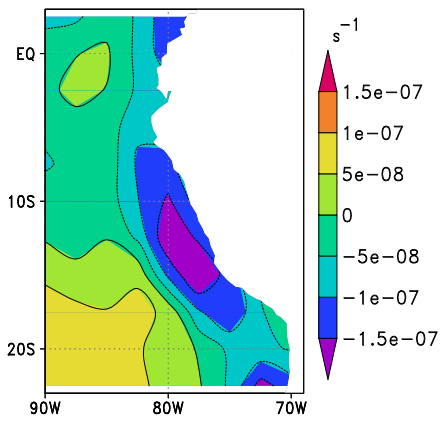

(i) Noviembre-Diciembre; Reanálisis

Figura 12: Promedios de rotacional de esfuerzo de viento, para los meses Julio - Agosto, Setiembre - Octubre, y Noviembre -Diciembre del año 2000. (a), (d), (g) datos de QuikSCAT; (b), (e), (h) datos de WRF; (c), (f), (i) datos de Reanálisis. Los colores indican la magnitud del rotacional, la que varía desde violeta $\left(-1.5 \times 10^{-7} s^{-1}\right)$ hasta rojo $\left(1.5 \times 10^{-7} \mathrm{~s}^{-1}\right)$, con intervalos de $-1.5 \times 10^{-7} \mathrm{~s}^{-1}$. Los valores positivos indican rotaciones anticiclónicas (sentido antihorario) y los negativos indican rotaciones clónicas (en sentido de las agujas del reloj). El comportamiento del rotacional juega un papel muy importante para la generación de afloramiento de aguas sub-superficiales. 